\title{
Expansão das Soluções de Sistemas Não Lineares no Espaço das Tunções Regradas \\ a Valores em Espaços de Banach
}

\section{Nelson G. Viloria Abreu}

TESE APRESENTADA

AO

INSTITUTO DE MATEMÁTICA E ESTATÍSTICA

DA

UNIVERSIDADE DE SÃO PAULO

PARA OBTENÇÃO DO GRAU

$\mathrm{DE}$

DOUTOR EM MATEMÁTICA APLICADA

Área de Concentração: Matemática Aplicada Orientador: Prof. Dr. Luciano Barbanti 
Expansão das Soluções de Sistemas Não Lineares

no Espaço das Funções Regradas a Valores em Espaços de Banach

Este exemplar corresponde à redação

final da tese, devidamente corrigida, apresentada por Nelson G. Viloria Abreu

e aprovada pela comissão julgadora.

São Paulo, 28 de Agosto de 1997.

Banca examinadora:

- Prof. Dr. Luciano Barbanti (Presidente) - IME - USP.

- Prof. Dr. Ricardo Bianconi - IME - USP.

- Prof. Dr. José Luis Boldrini - IMECC - UNICAMP.

- Prof. Dr. Ricardo Kubrusly - LNCC - CNPq.

- Prof. Dr. Roberto Moura Sales - EP - USP. 
A la mujer que amo.

A mis hijos queridos.

$\mathrm{Al}$ futuro que se convierte en presente: "El tiempo posible".

Dois horizontes fecham nossa vida:

Um horizonte, a saudade

Do que não há de voltar;

Outro horizonte, a esperança Dos tempos que hão de chegar;

No presente, sempre escuro,

Vive a alma ambiciosa

$\mathrm{Na}$ ilusão voluptuosa

Do passado e do futuro.

Machado de Assis. 


\section{Agradecimentos}

Quero expressar meu agradecimento ao Professor Luciano Barbanti por ter-me orientado na elaboração deste trabalho.

Também quero agradecer aos amigos, colegas e professores do IME, particularmente aos professores Chaim Hönig e Cyro Patarra.

Para finalizar, à CAPES e à ULA, entidades que facilitaram a realização desta tarefa. 


\section{Sumário}

Resumo i

Abstract

Introdução $\quad$ iii

1 Notações e resultados fundamentais 1

1.1 Funções regradas . . . . . . . . . . . . . . . . . . . . . . 2

1.2 Semivariação . . . . . . . . . . . . . . . . 6

1.3 Integral interior de Dushnik . . . . . . . . . . . . 7

1.4 Operadores m-lineares ..................... . . 11

2 Representação integral $\quad 12$

2.1 Extensão do conceito de semivariação . . . . . . . . . . . . . . . . 12 
2.2 Multi-integral interior de Dushnik . . . . . . . . . . . . . . 16

2.3 Teoremas de representação integral . . . . . . . . . . . . . . . . 20

2.4 Aproximação de operadores não lineares . . . . . . . . . . . . . . . . 31

3 Expansões para sistemas não lineares $\quad 34$

3.1 Representação em séries de Volterra . . . . . . . . . . . . . . . 35

3.2 O operador de Nemytskij . . . . . . . . . . . . . . . . . . . . . 39

3.3 O sistema não linear $(K) \ldots \ldots \ldots \ldots$. . . . . . . . . . 41

3.3.1 Existência de soluções . . . . . . . . . . . . . . . 42

3.3.2 Exemplo: um circuito não linear . . . . . . . . . . . . . . . 43

$\begin{array}{ll}\text { Bibliografia } & 49\end{array}$ 


\section{Resumo}

A teoria das equações do tipo $(K)$, no caso linear, tem sido e vem sendo bastante desenvolvida, principalmente por Hönig e seu grupo, desde a década de 70, estando relativamente completa em seus pontos fundamentais. Por outro lado, para sistemas não lineares (apesar do crescente uso de operadores de Volterra não lineares na Teoria do Controle e em geral em sistemas não lineares), temos apenas um resultado de existência, unicidade e dependência contínua de soluções dado por Hönig em 1985, para a equação

$$
x(t)+\int_{a}^{t} d_{s} K(t, s) f(s, x(s))=u(t), \quad t \in[a, b] .
$$

onde a não linearidade é dada pela $f$.

Neste trabalho expressamos a solução de $(K)$ em uma série do tipo de potências cujos termos são integrais de Stieltjes. Isto exigiu o desenvolvimento da teoria de representação integral do tipo Riesz para os operadores multilineares envolvidos naturalmente no problema. Além disso foi feita uma aplicação na teoria de circuitos não lineares. 


\section{Abstract}

The general theory for the $(K)$ Volterra-Stieltjes linear systems on Banach spaces -with both inputs and outputs being regulated functions- was well developed from the seventies, mainly by Hönig and his research group.

However, despite its increasing importance in control theory, we have for the nonlinear case only an existence and continuous dependence of (regulated) solutions theorem done by Hönig in 85 . He took on consideration the equation

$$
x(t)+\int_{a}^{t} d_{s} K(t, s) f(s, x(s))=u(t), \quad t \in[a, b] .
$$

in wich the nonlinearity was done by $f$.

In this work, we provide a power-series-like expression for the $(K)$ system outputs in terms of its inputs. For to do so, it was necessary to develop the whole representation theory (of the Riesz kind) for the multilinear operators involved into the problem.

It was done, finally, an example -a nonlinear system arising from the feed-back system theory for circuits- with the sake of to apply the results. 


\section{Introdução}

É crescente o interesse que se tem acerca dos sistemas de equações integrais a. valores num espaço de Banach. Normalmente, na literatura existente, o âmbito em que se configuram as questões são os espaços $L_{2}[a, b]$ e $C[a, b]$.

As equações integrais de Volterra-Stieljes do tipo $(K)$, são tratadas no espaço das funções regradas. E este espaço, apesar da evidente maior dificuldade de tratamento, é em varios contextos uma necessidade (às vezes não atendida, exatamente pelas dificuldades intrínsecas no mesmo, vide [5], [6] e [7]).

A teoria das equações do tipo $(K)$, no caso linear, tem sido e vem sendo bastante contemplada, principalmente por Hönig e seu grupo, desde a década de 70, estando relativamente completa em seus pontos fundamentais. Por outro lado, para sistemas não lineares (apesar do crescente uso de operadores de Volterra não lineares na Teoria do Contrôle e em geral nos sistemas não lineares, vide [4]) temos apenas um resultado de existência, unicidade de soluções e dependência contínua de soluções dado por Hönig em 1985, para a equação

$$
x(t)+\int_{a}^{t} d_{s} K(t, s) f(s, x(s))=u(t), \quad t \in[a, b] .
$$


onde a não linearidade é dada pela $f$.

Neste trabalho expressamos a solução de $\left(K^{\circ}\right)$ em uma série do tipo de potências cujos termos são integrais de Stieltjes o que exigiu o desenvolvi$\underline{\text { mento da teoria de representação integral do tipo Riesz para os operado- }}$ res multilineares envolvidos naturalmente no problema. Além disso é feita uma aplicação num modelo de circuitos não lineares conhecido na teoria de sistemas realimentados.

Para atingir este objetivo o presente trabalho foi esquematizado em três capítulos: o primeiro trata da linguagem fundamental na ligação das três noções essenciais no contexto de $(K)$ (funções regradas, semivariação e integral interior) e dos resultados básicos sobre operadores multilineares. O segundo capítulo versa sobre a representação integral com a respectiva generalização dos conceitos necessários no caso multilinear, obtendo-se assim um teorema do tipo Weierstrass como uma aplicação. O terceiro capítulo finaliza a questão com a representação de operadores em séries de Volterra e a expansão da solução do sistema $(K)$ como uma série de potências (com termos integrais). Um exemplo de um circuito elétrico modelado por tal equação é dado ao final.

NOTA EM RELAÇÃO A METODOLOGIA: Para destacar os resultados obtidos neste trabalho, devemos convencionar que só estes aparecem com sua respectiva prova. As proposições existentes na literatura são apenas citadas com sua referência correspondente. 


\section{Capítulo 1}

\section{Notações e resultados fundamentais}

Apresentamos neste capítulo os conceitos de funções regradas, semivariação e integral interior de Dushnik, básicos nos capítulos seguintes. Enunciamos as propriedades fundamentais referentes aos mesmos, as quais podem ser encontradas, em sua maioria, em [12].

Para finalizar o capítulo estabelecemos as notações, e fazemos um resumo das propriedades, das aplicações m-lineares em espaços de Banach. Uma referência clássica para este tema é [10]. Já para uma notação mais atualizada temos [4]. 


\subsection{Funções regradas}

Num belo artígo, primordialmente pedagógico, Berberian [2] fez uma apologia das funções regradas. Ele sugere que a teoria de funções regradas substitua a integral de Riemann no curso de Análise no começo do bacharelado e mesmo no curso de Análise Real do mestrado (como um instrutivo preludio à teoria de Lebesgue). Como as funções regradas são "pouco" utilizadas, temos procurado um defensor consagrado de seu uso (fora, é claro, do extenso trabalho de Hönig o qual é cotidianamente citado neste escrito). Não obstante, temos conferido sua utilização em áreas aplicadas tão diversas como Teoria de Populações Estruturadas $[7,6]$ e nos sistemas de realimentação [5].

Consideremos $[a, b] \subset \mathbb{R}$, um intervalo fechado, e $X, Y, W$ e $Z$ espaços de Banach.

DEFINIÇÃO 1.1.1. Uma partição de $[a, b]$ é um conjunto finito $P=\left\{t_{0}, \ldots, t_{n}\right\}$ com $a=t_{0}<\ldots<t_{n}=b$. Escrevemos $|P|=\max \left\{t_{r}-t_{r-1}: 1 \leq r \leq n(P)\right\}$, $n(P)=n$, e $P_{1} \leq P_{2}$ o fato de $P_{1} \subset P_{2}$. Denotamos por $\mathbb{P}[a, b]$ o conjunto de todas as partições de $[a, b]$.

DEFINIÇÃO 1.1.2. $x:[a, b] \rightarrow X$ é uma função escada sobre $[a, b]$ se existe uma partição $\left\{t_{0}, \ldots, t_{n}\right\}$ de $[a, b]$ tal que $x$ é constante em $\left(t_{r-1}, t_{r}\right)$, para todo $r$, $r=1, \ldots, n$. Denotamos por $E([a, b], X)$ o espaço das funções escada de $[a, b]$ em $X$.

DEFINIÇÃO 1.1.3. $x:[a, b] \rightarrow X$ é uma função regrada se $x$ só tem descontinuidades de primeira espécie, isto é, se 
(i) para todo $t \in[a, b)$ existe $x(t+)=\lim _{\tau \downarrow t} x(\tau) \quad e$

(ii) para todo $t \in(a, b]$ existe $x(t-)=\lim _{\tau \uparrow t} x(\tau)$.

Por $G([a, b], X)$ designamos o espaço das funções regradas de $[a, b]$ em $X$, munido da norma do sup.

ObSERVAÇÃo. É direto desta definição que $C([a, b], X) \subset G([a, b], X)$, i.e., que toda função contínua é regrada.

Outros espaços de funções a valores reais de uso freqüente (variação limitada, monótonas, Lipschitz, absolutamente contínuas, Darboux, com primitiva, etc) estão estreitamente ligados com o espaço das funções regradas como ilustra, esquemáticamente Muntean [20].

Muitas das propriedades mais importantes das funções regradas depreendem-se do seguinte

TeOrema 1.1.1. [12, Theorem I.3.1] Seja $x:[a, b] \rightarrow X$, então as propriedades seguintes são equivalentes:

(i) $x \in G([a, b], X)$.

(ii) $x$ é limite uniforme de funções escada.

(iii) Para todo $\epsilon>0$ existe $P \in \mathbb{P}[a, b]$ tal que $\omega_{P}(x)<\epsilon$, onde

$$
\omega_{P}(x)=\sup _{1 \leq r \leq n(P)} \sup _{t, s \in\left(t_{r-1}, t_{r}\right)}\|x(t)-x(s)\| .
$$

OBSERVAÇÃo. Este teorema estabelece que no espaço das funções limitadas, $\mathcal{B}([a, b], X)$, 
com a norma do sup, temos

$$
\overline{E([a, b], X)}=G([a, b], X) .
$$

OBSERVAÇÃo. Como corolário, da parte (iii) do teorema anterior, resulta que dada $x \in G([a, b], X)$, para todo $\epsilon>0$, os conjuntos

$$
\{t \in[a, b):\|x(t+)-x(t)\| \geq \epsilon\} \text { e }\{t \in(a, b]:\|x(t)-x(t-)\| \geq \epsilon\}
$$

são finitos. E portanto, o conjunto das descontinuidades de uma função regrada é enumerável.

Outra consequência, não menos importante, é o seguinte

TeOrema 1.1.2. [12, Theorem I.3.6] $G([a, b], X)$ munido da norma do sup é um espaço de Banach.

Para funções regradas, a relação entre derivação e integração esta dada pelo Teorema 1.1.3. [12, Theorem I.3.8] Sejam $x, y:[a, b] \longrightarrow X$. As propriedades seguintes são equivalentes:

(i) $x$ é regrada e y é uma primitiva de $x$.

(ii) $x$ é regrada e $y(t)=y(a)+\int_{a}^{t} x(s) d s$.

(iii) Para todo $t \in[a, b)$ existe $y_{+}^{\prime}=x(t+)$ e para todo $t \in(a, b]$ existe $y_{-}^{\prime}=x(t-)$.

DEFINIÇÃo 1.1.4. $x:[a, b] \rightarrow X$ é uma função regrada pela esquerda se

(i) $x(a)=0$

(ii) $x(t)=x(t-)$, para todo $t \in(a, b]$. 
OBSERVAÇÃo. [12, Theorem I.3.11] O espaço das funções regradas pela esquerda, $G^{-}([a, b], X)$, é um subespaço fechado de $G([a, b], X)$

DEFINIÇÃo 1.1.5. $x \in \Omega_{0}([a, b], X)$ se, e só se, para todo $\epsilon>0$ o conjunto $\{t \in[a, b]:\|x(t)\| \geq \epsilon\} \quad$ é finito.

OBSERvaÇÃo. Da definição anterior temos que $\Omega_{0}([a, b], X)$ é um subespaço fechado de $G([a, b], X)$. Além disso, em [12] caracteriza-se $\Omega_{0}([a, b], X)$ e mostra-se que

$$
G([a, b], X)=G^{-}([a, b], X) \oplus \Omega_{0}([a, b], X)
$$

DEFINIÇÃO 1.1.6. $x:[a, b] \rightarrow L(W, X)$ é uma função simplesmente regrada se, para todo $w \in W$, a função

$$
\begin{aligned}
x \cdot w:[a, b] & \longrightarrow X \\
t & \longmapsto x(t) w
\end{aligned}
$$

é regrada. Escrevemos $x \in G^{\sigma}([a, b], L(W, X))$.

OBservaÇÃo. Do Teorema de Banach-Steinhaus, para todo $t \in[a, b)$ (respectivamente $t \in(a, b])$, existe $x(t \dot{+})(x(t \dot{-})) \in L(W, X)$ tal que

$$
\lim _{t_{n} \downarrow t} x\left(t_{n}\right) w=x(t \dot{+}) w\left(\lim _{t_{n} \uparrow t} x\left(t_{n}\right) w=x(t \dot{-}) w\right) .
$$

Teorema 1.1.4. [1, Props. 1.9 e 1.11] $G^{\sigma}([a, b], L(X, W))$ é um espaço de Banach quando munido da norma do sup e $G^{\sigma}([a, b], L(X, W)) \subset \mathcal{B}([a, b], L(X, W))$. Além disso, $G([a, b], L(W, X)) \subset G^{\sigma}([a, b], L(W, X))$ e a igualdade vale se, e só se, $\operatorname{dim} W<\infty$. 


\subsection{Semivariação}

A seguir damos o conceito de semivariação, cuja noção foi criada em 1936 por Gowurin [9]. Uma aplicação, onde o conceito joga um papel primordial, foi dada em 1995 por Diekmann(e outros) [7]

DEFINIÇÃo 1.2.1. Se $\alpha:[a, b] \longrightarrow L(X, Y)$, a semivariação de Vitali de $\alpha$ em $[a, b]$ é dada por

$$
S V[\alpha]=S V_{[a, b]}[\alpha]=\sup _{P} S V_{P}[\alpha]
$$

onde

$$
S V_{P}\left[\stackrel{\alpha}{K_{1}^{\prime}}\right]=\sup _{\left\|x_{i}\right\| \leq 1}\left\{\left\|\sum_{i=1}^{n(P)}\left[\alpha\left(t_{i}\right)-\alpha\left(t_{i-1}\right)\right]\left(x_{i}\right)\right\|: x_{i} \in X\right\} .
$$

Se $S V[\alpha]<\infty$, dizemos que $\alpha$ é de semivariação de Vitali limitada e escrevemos $\alpha \in S V([a, b], L(X, Y))$. Se, além disso, $\alpha(a)=0$, escrevemos:

$$
\alpha \in S V_{0}([a, b], L(X, Y))
$$

Para $[c, d] \subset[a, b]$ definimos

$$
S V_{(c, d]}[\alpha]=\lim _{\tau \downarrow c} S V_{[\tau, d]}[\alpha]
$$

e do modo análogo $S V_{[c, d)}[\alpha]$.

Teorema 1.2.1. [11, I.1.2 e I.3.1] Seja $\alpha \in S V([a, b], L(X, Y))$. Então,

(a) $S e[c, d] \subset[a, b]$, então $S V_{[c, d]}[\alpha] \leq S V_{[a, b]}[\alpha]$.

(b) Se $c \in[a, b]$, então $S^{\prime} V_{[a, b]}[\alpha] \leq S V_{[a, c]}[\alpha]+S V_{[c, b]}[\alpha]$.

(c) $\|\alpha(t)\| \leq\|\alpha(a)\|+S V_{[a, b]}[\alpha]$. 
(d) A função $\alpha \longmapsto S V[\alpha]$ é uma seminorma sobre $S V([a, b], L(X, Y))$.

TeOrema 1.2.2. [11, I.1.3] $S V_{0}([a, b], L(X, Y))$ é um espaço de Banach quando munido da norma $\alpha \longmapsto S V[\alpha]$.

DEFINIÇÃo 1.2.2. Se $\alpha:[a, b] \longrightarrow X$, a variação de Vitali de $\alpha$ em $[a, b]$ é dada por

$$
V[\alpha]=V_{[a, b]}[\alpha]=\sup _{P} V_{P}\left[K^{\prime}\right]
$$

onde

$$
V_{P}\left[K^{i}\right]=\sum_{i=1}^{n(P)}\left\|\alpha\left(t_{i}\right)-\alpha\left(t_{i-1}\right)\right\| .
$$

Se $V[\alpha]<\infty$, dizemos que $\alpha$ é uma função de Variação (de Vitali) limitada e denotamos isto por $\alpha \in B V([a, b], X)$.

As funções de variação limitada também estão contidas no espaço das funções regradas, como vemos no seguinte

Teorema 1.2.3. [11, C5] $B V([a, b], X) \subset G([a, b], X)$.

O conceito de semivariação é uma extensão do conceito de variação segundo mostra o

Teorema 1.2.4. [14, 1.8] Se $\alpha \in B V([a, b], L(X, Y))$, então $\alpha \in S V([a, b], L(X, Y))$ e $S V[\alpha] \leq V[\alpha]$. A igualdade é válida se, e só se, $\operatorname{dim} Y<\infty$.

\subsection{Integral interior de Dushnik}

Nesta seção apresentamos o conceito de integral interior o qual foi definido pela primeira vez em 1920 por Pollard(vide [14]) e redescoberto em 1931 por Dush- 
nik(vide [14]). Mas eles não estabeleceram resultados muito significantes. Já em 1934, Kaltenborn [17] utilizou este conceito para representar os elementos de $L(G([a, b], \mathbb{R}), \mathbb{R})$. Este resultado foi generalizado a $L(G([a, b], X), Y)$ em 1975 por Hönig [12] e estendido à $L_{m}(G([a, b], X), Y)$, neste trabalho.

DEFINIÇÃO 1.3.1. Sejam e, $\left(e_{P}\right)_{P \in P}$ pontos de um espaço topológico E, escrevemos $e=\lim _{P} e_{P}$ quando, para toda vizinhança $V$ de ex existe $P_{V} \in \mathbb{P}$ tal que

$$
P \geq P_{V} \Longrightarrow e_{P} \in V \text {. }
$$

DEFINIÇÃO 1.3.2. Sejam $x:[a, b] \longrightarrow X$ e $\alpha:[a, b] \longrightarrow L(X, Y)$ definimos $a$ integral interior de Dushnik por

$$
\int_{a}^{b} d \alpha(t) x(t)=\lim _{P \in P} \sum_{i=1}^{n(P)}\left[\alpha\left(t_{i}\right)-\alpha\left(t_{i-1}\right)\right] x\left(\xi_{i}\right), \quad \xi_{i} \in\left(t_{i-1}, t_{i}\right),
$$

e a integral de Riemann-Stieltjes por

$$
\left(R-S^{\prime}\right) \int_{a}^{b} d \alpha(t) x(t)=\lim _{|P| \rightarrow 0} \sum_{i=1}^{n(P)}\left[\alpha\left(t_{i}\right)-\alpha\left(t_{i-1}\right)\right] x\left(\xi_{i}\right), \quad \xi_{i} \in\left[t_{i-1}, t_{i}\right],
$$

quando os limites envolvidos existem.

A integral interior de Dushnik generaliza a de Riemann-Stieltjes de acôrdo com o seguinte

Teorema 1.3.1. [12, Theorems I.1.1 e I.1.2] Se existe $(R-S) \int_{a}^{b} d \alpha(t) x(t)$, então existe $\int_{a}^{b} d \alpha(t) x(t)$. Além disso, se $x$ e a são duas funções limitadas que não têm descontinuidades comuns e a integral interior de Dushnik existe, então a de RiemannStieltjes também. Nos dois casos, os valores das duas integrais coincidem. 
ExeMplo 1. Considere $c \in(0,1), \alpha=\lambda_{[c, 1]}$ e $x=\gamma_{(c, 1]}$. Entĩo,

$$
\int_{0}^{1} d \alpha(t) x(t)=1
$$

enquanto que a integral correspondente de Riemann-Stieltjes não existe, pois $\alpha$ e $x$ têm uma descontinuidade comum em c.

Teorema 1.3.2. Dado $c \in(a, b), \int_{a}^{b} d \alpha(t) x(t)$ existe se, e só se, $\int_{a}^{c} d \alpha(t) x(t) e$ $\int_{c}^{b} d \alpha(t) x(t)$ existem. Neste caso, temos que

$$
\int_{a}^{b} d \alpha(t) x(t)=\int_{a}^{c} d \alpha(t) x(t)+\int_{c}^{b} d \alpha(t) x(t)
$$

Lembremos que esta propiedade não se verifica com a integral de Riemann-Stieltjes como vemos no

ExEMPLO 2. No exemplo 1, temos que

$$
(R-S) \int_{0}^{c} d \alpha(t) x(t)=0 \quad e \quad(R-S) \int_{c}^{1} d \alpha(t) x(t)=1
$$

Mas, $(R-S) \int_{0}^{1} d \alpha(t) x(t)$ não existe.

Acontece o contrário com a fórmula de integração por partes. No próximo exemplo vemos como a interior de Dushnik não a verifica.

Exemplo 3. No exemplo 1, definimos

$$
x_{c}(t)= \begin{cases}x(t), & \text { se } t \neq c \\ c, & \text { se } t=c\end{cases}
$$


Assim,

$$
\int_{0}^{1} d \alpha(t) x_{c}(t)=0, \quad \int_{0}^{1} \alpha(t) d x_{c}(t)=1-c
$$

$e$

$$
\alpha(1) x_{c}(1)-\alpha(0) x_{c}(0)=1
$$

Embora a integral interior de Dushnik não verifique a fórmula de integração por partes, existe uma integral associada a esta que dá uma fórmula modificada:

$$
\int_{a}^{b} d \alpha(t) x(t)+(Y) \int_{a}^{b} \alpha(t) d x(t)=\alpha(b) x(b)-\alpha(a) x(a)
$$

onde:

$$
\begin{aligned}
(Y) \int_{a}^{b} \alpha(t) d x(t)= & \lim _{P \in P} \sum_{i=1}^{n(P)}\left\{\alpha\left(t_{i-1}\right)\left[x\left(t_{i-1} \dot{+}\right)-x\left(t_{i-1}\right)\right]+\alpha\left(\xi_{i}\right)\left[x\left(t_{i} \dot{-}\right)-x\left(t_{i-1} \dot{+}\right)\right]\right. \\
& \left.+\alpha\left(t_{i}\right)\left[x\left(t_{i}\right)-x\left(t_{i} \dot{-}\right)\right]\right\}, \quad \xi_{i} \in\left(t_{i}-1, t_{i}\right)
\end{aligned}
$$

é a integral de Young.

Para finalizar esta seção, pela definição da integral interior de Dushnik, temos que verificam-se os enunciados habituais de bilinearidade.

Teorema 1.3.3. Se $\alpha_{1}, \alpha_{2}:[a, b] \longrightarrow L(X, Y)$ e $x_{1}, x_{2}:[a, b] \longrightarrow X$ são tais que as integrais envolvidas existem. Então valem as igualdades:

$$
\begin{aligned}
\int_{a}^{b} d \alpha(t)\left(x_{1}+x_{2}\right)(t) & =\int_{a}^{b} d \alpha(t) x_{1}(t)+\int_{a}^{b} d \alpha(t) x_{2}(t) \\
\int_{a}^{b} d(\alpha+\beta)(t) x(t) & =\int_{a}^{b} d \alpha(t) x(t)+\int_{a}^{b} d \beta(t) x(t) . \\
\int_{a}^{b} d \alpha(t)(\lambda x)(t) & =\int_{a}^{b} d(\lambda \alpha)(t) x(t)=\lambda \int_{a}^{b} d \alpha(t) x(t)
\end{aligned}
$$




\subsection{Operadores m-lineares}

Sejam $X_{1}, \ldots, X_{m}$ e $Y$ espaços de Banach, e consideremos $\prod_{r=1}^{m} X_{r}$ com a norma produto usual induzida pelas normas sobre os $X_{r}, r=1, \ldots, m$, i.e., $\|x\|=\sup \left\|x_{r}\right\|$, se $x=\left(x_{1}, \ldots, x_{m}\right) \in \prod_{r=1}^{m} X_{r}$.

DEFINIÇÃo 1.4.1. Uma aplicação $\Lambda: \prod_{r=1}^{m} X_{r} \longrightarrow Y$ é $\underline{\text { m-linear }}$ (ou $\underline{\text { multilinear }}$ ) se

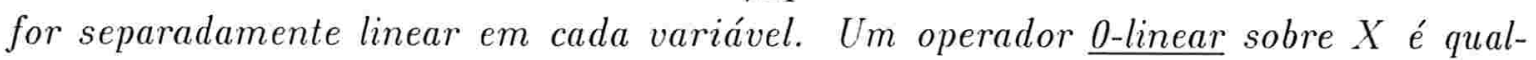
quer aplicação constante sobre $X$, e identificamos os operadores 0-lineares com a sua imagem. A aplicação $\Lambda$ é dita simétrica se $\Lambda\left(x_{1}, \ldots, x_{m}\right)=\Lambda\left(x_{l(1)}, \ldots, x_{l(m)}\right)$ para qualquer permutação $l$ dos inteiros $\{1, \ldots, m\}$.

DEFINIÇÃo 1.4.2. A aplicação m-linear $\Lambda: \prod_{r=1}^{m} X_{r} \longrightarrow Y$ é limitada se existe $M \geq 0$ tal que $\left.\left\|\Lambda\left(x_{1}, \ldots, x_{m}\right)\right\| \leq M\left\|x_{1}\right\| \cdots\left\|x_{m}\right\|\right)$.

Teorema 1.4.1. [4, Theorem 1.17] A aplicação m-linear $\Lambda$ é contínua se, e só se, é limitada.

Teorema 1.4.2. [4, Theorem 1.13] Para todo $k=1, \ldots, m$, temos que

$$
L\left(X_{1}, \ldots, X_{m} ; Y\right) \simeq L\left(X_{1}, \ldots, X_{k} ; L\left(X_{k+1}, \ldots, X_{m} ; Y\right)\right)
$$

DEFINIÇÃo 1.4.3. Um operador polinomial de grau $m$ sobre um espaço de Banach $X$ é uma aplicação $P: X \longrightarrow X$ da forma

$$
P x=L_{0}+L_{1} x+\ldots+L_{m}(x, \ldots, x)
$$

onde $L_{0} \in X$ e os $L_{r}$ são aplicações r-lineares simétricas (que são chamadas de operadores polinomiais homogêneos de grau r ou simplesmente operadores r-potência).

Usando a nótação $L_{r}(x, \ldots, x)=L_{r}[x]^{r}$, podemos expressar o operador $P$ na forma

$$
P x=L_{0}+L_{1}[x]+\ldots+L_{m}[x]^{m} .
$$




\section{Capítulo 2}

\section{Representação integral}

Neste capítulo caracterizamos a representação integral de operadores multilineares em $G^{-}([a, b], X)$. Para isto foi necessário generalizar os conceitos de semivariação e integral interior de Dushnik para aplicações definidas num m-bloco. No final do capítulo mostramos um teorema de aproximação de Weierstrass, como uma aplicação desta teoria de representação integral múltipla.

\subsection{Extensão do conceito de semivariação}

No caso multilinear o conceito de semivariação, análogamente ao caso da variação, bifurca-se em dois sentidos: o sentido de Vitali (definição 2.1.4) e o sentido de Fréchet (definição 2.1.5). A TABELA 1 esquematiza este desdobramento. 
DEFINIÇÃo 2.1.1. Uma partição de um m-bloco, $\prod_{r=1}^{m}\left[a_{r}, b_{r}\right] \subset \mathbb{R}^{m}$, é um conjunto finito do tipo $P=\prod_{r=1}^{m} P_{r}$, onde $P_{r} \in \mathbb{P}\left[a_{r}, b_{r}\right]$. Os pontos de $P_{r}$ satisfazem

$$
a_{r}=t_{0(r)}<\ldots<t_{n(r)}=b_{r} .
$$

Fazemos $n(P)=\prod_{r=1}^{m} n\left(P_{r}\right)$ e $|P|=\prod_{r=1}^{m}\left|P_{\mathrm{r}}\right|$. O conjunto das partições do $m$-bloco é denotado por $\mathbb{P}\left(\prod_{r=1}^{m}\left[a_{r}, b_{r}\right]\right)$.

DEFINIÇÃo 2.1.2. Sejam $z: \prod_{r=1}^{m}\left[a_{r}, b_{r}\right] \longrightarrow Z \quad$ e $P=\prod_{r=1}^{m} P_{r}$, onde $P_{r} \in \mathbb{P}\left[a_{r}, b_{r}\right]$ e $a_{r}=t_{0(r)}<\ldots<t_{n(r)}=b_{r}$. Fixando $r$, para um inteiro $i(r), 1 \leq i(r) \leq n$, definimos:

Se $m=1$,

$$
\Delta_{i} z=z\left(t_{i}\right)-z\left(t_{i-1}\right)
$$

Se $m \geq 2$,

$$
\Delta_{i(r)} z: \prod_{j=1}^{r-1}\left[a_{j}, b_{j}\right] \times \prod_{j=r+1}^{m}\left[a_{j}, b_{j}\right] \longrightarrow Z
$$

por

$$
\begin{aligned}
\left(\Delta_{i(r)} z\right)\left(s_{1}, \ldots, s_{r-1}, s_{r+1}, \ldots, s_{m}\right)= & z\left(s_{1}, \ldots, s_{r-1}, t_{i(r)}, s_{r+1}, \ldots, s_{m}\right) \\
& -z\left(s_{1}, \ldots, s_{r-1}, t_{i(r)-1}, s_{r+1}, \ldots, s_{m}\right) .
\end{aligned}
$$

Considerando $q, 1 \leq q \leq m$, denotamos por $\Delta_{i(1)} \Delta_{i(2)} \ldots \Delta_{i(q)} z$ o cálculo $\Delta_{i(1)}\left(\Delta_{i(2)}\left(\ldots \Delta_{i(q)} z\right) \ldots\right)\left(s_{q+1}, \ldots, s_{m}\right)$.

DEFINIÇÃo 2.1.3. Seja $K: \prod_{r=1}^{m}\left[a_{r}, b_{r}\right] \longrightarrow Z$. A variação de Vitali de $K$ em $\prod_{r=1}^{m}\left[a_{r}, b_{r}\right]$ é dada por

$$
V[K]=\sup _{P} V_{P}[K],
$$


onde,

$$
V_{P}\left[K^{\prime}\right]=\sum_{i(1), \ldots i(m)}^{n(P)}\left\|\Delta_{i(1)} \ldots \Delta_{i(m)} K^{\prime}\right\|
$$

com $P \in \mathbb{P}\left(\prod_{r=1}^{m}\left[a_{r}, b_{r}\right]\right)$

Se $V[K]<\infty$, dizemos que $K$ é de variação limitada em $\prod_{r=1}^{m}\left[a_{r}, b_{r}\right]$ e escrevemos $\kappa \in B V\left(\prod_{r=1}^{m}\left[a_{r}, b_{r}\right], Z\right)$.

DEFINIÇÃo 2.1.4. Se K: $\prod_{r=1}^{m}\left[a_{r}, b_{r}\right] \longrightarrow L(X, Y)$, a semivariação de Vitali de $K$ em $\prod_{r=1}^{m}\left[a_{r}, b_{r}\right]$ é definida por

$$
S V\left[K^{-}\right]=\sup _{P} S V_{P}\left[K^{\prime}\right]
$$

onde

$$
S V_{P}[K]=\sup _{\left\|x_{i(1) \ldots i(m)}\right\| \leq 1}\left\{\left\|\sum_{i(1), \ldots, i(m)}^{n(P)} \Delta_{i(1)} \ldots \Delta_{i(m)} K\left(x_{i(1) \ldots i(m)}\right)\right\|: x_{i(1) \ldots i(m)} \in X\right\},
$$

com $P \in \mathbb{P}\left(\prod_{r=1}^{m}\left[a_{r}, b_{r}\right]\right)$

Se $S V[K]<\infty$, dizemos que $K$ é de semivariação de Vitali limitada e escrevemos $K \in S V\left(\prod_{r=1}^{m}\left[a_{r}, b_{r}\right], L(X, Y)\right)$.

OBSERVAÇÃo. A função $K \longmapsto S V[K]$ é uma seminorma sobre o espaço

$$
S V\left(\prod_{r=1}^{m}\left[a_{r}, b_{r}\right], L(X, Y)\right)
$$

Teorema 2.1.1. $B V\left(\prod_{r=1}^{m}\left[a_{r}, b_{r}\right], L(X, Y)\right) \subset S V\left(\prod_{r=1}^{m}\left[a_{r}, b_{r}\right], L(X, Y)\right) \quad e s e$ $K \in B V\left(\prod_{r=1}^{m}\left[a_{r}, b_{r}\right], L(X, Y)\right)$, então $\quad S V\left[K^{\prime}\right] \leq V[K]$. 
Prova: Dadas $K \in B V\left(\prod_{r=1}^{m}\left[a_{r}, b_{r}\right], L(X . Y)\right), P=\prod_{r=1}^{m} P_{r} \operatorname{com} P_{r} \in \mathbb{P}\left[a_{r}, b_{r}\right]$ e $x_{i(1) \ldots i(m)} \in X, \quad$ com $1 \leq i(r) \leq n\left(P_{r}\right), r=1, \ldots, m$, tal que $\left\|x_{i(1) \ldots i(m)}\right\| \leq 1$, temos que

$$
\left\|\sum_{i(1), \ldots, i(m)}^{n(P)} \Delta_{i(1)} \ldots \Delta_{i(m)} K\left(x_{i(1) \ldots i(m)}\right)\right\| \leq \sum_{i(1), \ldots, i(m)}^{n(P)}\left\|\Delta_{i(1)} \ldots \Delta_{i(m)} K\right\| \leq V[K] .
$$

Logo $S V\left[K^{\prime}\right] \leq V\left[K^{\prime}\right]$

O conceito de variação de Fréchet foi usado por ele mesmo em [8] para representar funcionais bilineares contínuos sobre $C([a, b], \mathbb{R})$. Tendo em vista a definição de semivariação obtemos a seguinte

DEFINIÇÃo 2.1.5. Para $K: \prod_{r=1}^{m}\left[a_{r}, b_{r}\right] \longrightarrow L\left(X_{1}, \ldots, X_{m} ; Y\right)$ a semivariação de Fréchet de $K$ em $\prod_{r=1}^{m}\left[a_{r}, b_{r}\right]$ é dada por

$$
S F[K]=\sup _{P} S F_{P}\left[K^{\prime}\right]
$$

onde

$$
S F_{P}[K]=\sup _{\left\|x_{i(r)}\right\| \leq 1}\left\{\left\|\sum_{i(1), \ldots, i(m)}^{n(P)} \Delta_{i(1)} \ldots \Delta_{i(m)} K\left(x_{i(1)}, \ldots, x_{i(m)}\right)\right\|: x_{i(r)} \in X_{r}\right\},
$$

com $P \in \mathbb{P}\left(\prod_{r=1}^{m}\left[a_{r}, b_{r}\right]\right)$.

Se $S F[K]<\infty$, dizemos que $K$ é de semivariação de Fréchet limitada e escreve$\operatorname{mos} K \in S F\left(\prod_{r=1}^{m}\left[a_{r}, b_{r}\right], L\left(X_{1}, \ldots, X_{m} ; Y\right)\right)$.

OBSERVAÇÃo. A função $K \longmapsto S F[K]$ é uma seminorma sobre o espaço

$$
S F\left(\prod_{r=1}^{m}\left[a_{r}, b_{r}\right], L(X, Y)\right) \text {. }
$$


De forma análoga ao teorema 2.2.1, mostra-se o seguinte

TEOREMA 2.1.2. $B V\left(\prod_{r=1}^{m}\left[a_{r}, b_{r}\right], L\left(X_{1}, \ldots, X_{m} ; Y\right)\right) \subset S F\left(\prod_{r=1}^{m}\left[a_{r}, b_{r}\right], L\left(X_{1}, \ldots, X_{m} ; Y\right)\right)$ Além disso, se $K \in B V\left(\prod_{r=1}^{m}\left[a_{r}, b_{r}\right], L\left(X_{1}, \ldots, X_{m} ; Y\right)\right)$, então $S F\left[K^{\prime}\right] \leq V\left[K^{\prime}\right]$.

Para finalizar esta seção, mostramos uma tabela que esquematiza as diferentes definições de (semi)variação tratadas:

\begin{tabular}{|c|c|c|c|c|c|}
\hline \multicolumn{6}{|c|}{$K: \prod_{r=1}^{m}\left[a_{r}, b_{r}\right] \longrightarrow Z$} \\
\hline \hline${ }_{m}^{Z}$ & $X$ & $L(X ; Y)$ & $L\left(X_{1}, X_{2} ; Y\right)$ & $\cdots$ & $L\left(X_{1}, \ldots, X_{m} ; Y\right)$ \\
\hline 1 & $B V$ & $S V$ & $\times$ & $\cdots$ & $\times$ \\
\hline 2 & $B V$ & $S V$ & $S F$ & & $\times$ \\
\hline$\vdots$ & $\vdots$ & $\vdots$ & $\vdots$ & $\ddots$ & $\vdots$ \\
\hline$N$ & $B V$ & $S V$ & $\cdots$ & & $S F$ \\
\hline
\end{tabular}

TABELA 1

\subsection{Multi-integral interior de Dushnik}

A integral interior múltipla foi utilizada por Prandini [23], para mostrar o teorema de representação integral para operadores bilineares sobre espaços de funções regradas. Aqui estendemos tal conceito para o caso m-dimensional e estabelecemos uma condição suficiente para sua existência. 
DEFINIÇÃO 2.2.1. Sejam $K^{\prime}: \prod_{r=1}^{m}\left[a_{r}, b_{r}\right] \longrightarrow L\left(X_{1}, \ldots, X_{m} ; Y\right)$ e $x_{r}:\left[a_{r}, b_{r}\right] \longrightarrow X_{r}$, $r=1, \ldots, m$. Se existe $\lim _{P \in P} \sigma_{P}$, onde

$$
\sigma_{P}=\sum_{i(1), \ldots, i(m)}^{n(P)} \Delta_{i(1)} \ldots \Delta_{i(m)} K\left(x_{1}\left(\xi_{i(1)}\right), \ldots, x_{m}\left(\xi_{i(m)}\right)\right),
$$

com $\xi_{i(r)} \in\left(t_{i(r)-1,3} t_{i(r)}\right) \quad e \quad \mathbb{P}=\mathbb{P}\left(\prod_{r=1}^{m}\left[a_{r}, b_{r}\right]\right)$, este limite é chamado de integral interior de Dushnik de $x=\left(x_{1}, \ldots, x_{m}\right)$ em relação a $K$ e denota-se por

$$
\int_{a_{1}}^{b_{1}} \ldots \int_{a_{m}}^{b_{m}} d_{s_{1} \ldots s_{m}} K\left(s_{1}, \ldots, s_{m}\right)\left(x_{1}\left(s_{1}\right), \ldots, x_{m}\left(s_{m}\right)\right) .
$$

A seguir damos, entre outras coisas, uma condição suficiente para a existência da integral.

LEMA 2.2.1. Se $K \in S F\left(\prod_{r=1}^{m}\left[a_{r}, b_{r}\right], L\left(X_{1}, \ldots, X_{m} ; Y\right)\right)$ e $x_{r} \in G\left(\left[a_{r}, b_{r}\right], X_{r}\right)$, $r=1, \ldots, m$. Então,

(i) existe $\Lambda_{K} x=\int_{a_{1}}^{b_{1}} \cdots \int_{a_{m}}^{b_{m}} d_{s_{1} \ldots s_{m}} K\left(s_{1}, \ldots, s_{m}\right)\left(x_{1}\left(s_{1}\right), \ldots, x_{m}\left(s_{m}\right)\right)$,

(ii) $\Lambda_{K}$ é m-linear,

iii) $\left\|\Lambda_{K} x\right\| \leq S F\left[K^{\prime}\right]\left\|x_{1}\right\| \cdots\left\|x_{m}\right\|$,

(iv) se $x_{r} \in \Omega_{0}\left(\left[a_{r}, b_{r}\right], X_{r}\right)$ para algum $r, r=1, \ldots, m$, então $\Lambda_{K} x=0$.

Prova: Se $x_{r}=0$, para algum $r$, ou $K=0$, tudo se verifica. Assim, considere$\operatorname{mos} x_{r} \neq 0, \quad r=1, \ldots, m$, e $K \neq 0$.

(i) Vamos verificar que o critério de Cauchy se cumpre. 
Seja $\epsilon>0$. Para todo $r, r=1, \ldots, m$, (vide teorema 1.1.1) existe $P_{r}(\epsilon) \in \mathbb{P}\left[a_{r}, b_{r}\right]$ tal que

$$
\omega_{P_{r}(\epsilon)}\left(x_{r}\right)<\frac{\epsilon\left\|x_{r}\right\|}{2 S F\left[K^{\prime}\right]\left\|x_{1}\right\| \cdots\left\|x_{m}\right\|} .
$$

Se $P \geq P(\epsilon)=\prod_{r=1}^{m} P_{r}(\epsilon)$, podemos obter $P$ a partir de $P(\epsilon)$ intercalando um número finito de pontos nas partições $P_{r}(\epsilon)$. Indutivamente ficamos reduzidos ao caso no qual $P$ é obtido inserindo-se um ponto em alguma partição $P_{k}(\epsilon)$, para algum $k, \mathrm{k}=1, \ldots, \mathrm{m}$. Seja $\mathcal{O}$, o ponto considerado, em algum intervalo de $P_{k}(\epsilon)$. Assim,

$$
\begin{aligned}
\sigma_{P}-\sigma_{P(\epsilon)}= & \sum_{i(1), \ldots, i(m)}^{n(P)} \Delta_{i(1)} \ldots \Delta_{i(m)} K\left(x_{1}\left(\xi_{i(1)}\right), \ldots, x_{k-1}\left(\xi_{i(k-1)}\right), x_{k}\left(\xi_{i(k)}\right)-x_{k}\left(\xi_{\mathcal{O}}\right),\right. \\
& \left.+\sum_{k+1}\left(\xi_{i(k+1)}\right), \ldots, x_{m}\left(\xi_{i(m)}\right)\right) \\
& \sum_{i(1), \ldots, i(m)}^{n(P)} \Delta_{i(1)} \ldots \Delta_{i(m)} K\left(x_{1}\left(\xi_{i(1)}\right), \ldots, x_{k-1}\left(\xi_{i(k-1)}\right), x_{k}\left(\xi_{\mathcal{O}}\right)-x_{k}\left(\xi_{i(k)-1}\right),\right. \\
= & \sum_{i(1), \ldots, i(m)}^{n(P)} \Delta_{i(1)} \ldots \Delta_{i(m)} K\left(\frac{x_{1}\left(\xi_{i(1)}\right)}{\left\|x_{1}\right\|} \ldots,\right. \\
& \left.+\frac{2 S F[K]\left\|x_{1}\right\| \ldots\left\|x_{m}\right\|}{\epsilon\left\|x_{k}\right\|}\left(x_{k}\left(\xi_{i(k)}\right)-x_{k}\left(\xi_{\mathcal{O}}\right)\right), \ldots, \frac{x_{m}\left(\xi_{i(m)}\right)}{\left\|x_{m}\right\|}\right) \frac{\epsilon}{2 S F\left[K^{\prime}\right]} \\
& \left.\quad \frac{2 S F\left[K^{\prime}\right]\left\|x_{1}\right\| \ldots\left\|x_{m}\right\|}{\epsilon\left\|x_{k}\right\|}\left(x_{k}\left(\xi_{\mathcal{O}}\right)-x_{k}\left(\xi_{i(k)-1}\right)\right), \ldots, \frac{x_{m}\left(\xi_{i(m)}\right)}{\left\|x_{m}\right\|}\right) \frac{\epsilon}{2 S F\left[K^{\prime}\right]}
\end{aligned}
$$

Logo,

$$
\left\|\sigma_{P}-\sigma_{P(\epsilon)}\right\| \leq S F[K] \frac{\epsilon}{2 S F\left[K^{\prime}\right]}+S^{\prime} F[K] \frac{\epsilon}{2 S^{\prime} F\left[K^{\prime}\right]}=\frac{\epsilon}{2}+\frac{\epsilon}{2}=\epsilon
$$


E portanto,

$$
P, \bar{P} \geq P(\epsilon) \Longrightarrow\left\|\sigma_{P}-\sigma_{P}\right\| \leq \epsilon
$$

(ii) É conseqüência direta da definição da integral.

(iii) Para qualquer $P \in \mathbb{P}\left(\prod_{r=1}^{m}\left[a_{r}, b_{r}\right]\right)$ temos

$$
\begin{aligned}
\left\|\sigma_{P}\right\| & =\left\|\sum_{i(1), \ldots, i(m)}^{n(P)} \Delta_{i(1)} \ldots \Delta_{i(m)} K\left(\frac{x_{1}\left(\xi_{i(1)}\right)}{\left\|x_{1}\right\|}, \ldots, \frac{x_{m}\left(\xi_{i(m)}\right)}{\left\|x_{m}\right\|}\right)\right\| \cdot\left\|x_{1}\right\| \cdots\left\|x_{m}\right\| \\
& \leq S F[K]\left\|x_{1}\right\| \cdots\left\|x_{m}\right\| .
\end{aligned}
$$

Assim, passando ao limite, resulta

$$
\left\|\Lambda_{K} x\right\| \leq S F\left[K^{\prime}\right]\left\|x_{1}\right\| \cdots\left\|x_{m}\right\|
$$

(iv) Sem perda de generalidade, suponha que $x_{1} \in \Omega_{0}\left(\left[a_{1}, b_{1}\right], X_{1}\right)$. Então, da definição de $\Omega_{0}$, para todo $\epsilon>0$ existe $P_{1}(\epsilon) \in \mathbb{P}\left[a_{1}, b_{1}\right]$ tal que

$$
\left\{t \in\left[a_{1}, b_{1}\right]:\left\|x_{1}(t)\right\| \geq \frac{\epsilon}{S F\left[K^{\prime}\right]\left\|x_{2}\right\| \cdots\left\|x_{m}\right\|}\right\} \subset P_{1}(\epsilon) .
$$

Donde, se $P=\prod_{r=1}^{m} P_{r}$ com $P_{1} \geq P_{1}(\epsilon)$ e as outras partições são quaisquer, temos que

$$
\begin{aligned}
\left\|\sigma_{P}\right\|= & \| \sum_{i(1), \ldots, i(m)}^{n(P)} \Delta_{i(1)} \ldots \Delta_{i(m)} K\left(\frac{S F[K]\left\|x_{2}\right\| \cdots \mid x_{m} \|}{\epsilon} x_{1}\left(\xi_{i(1)}\right),\right. \\
& \left.\frac{x_{2}\left(\xi_{i(2)}\right)}{\left\|x_{2}\right\|}, \ldots, \frac{x_{m}\left(\xi_{i(m))}\right.}{\left\|x_{m}\right\|}\right) \| \frac{\epsilon}{S F[K]} \\
< & \epsilon .
\end{aligned}
$$

Logo, $\Lambda_{K} x=0$. 
Teorema 2.2.2. [2.3, Teorema 4.3] Sejam $x_{r} \in G\left(\left[a_{r}, b_{r}\right], X_{r}\right)$, para $r, r=1, \ldots, m$ e $K \in S F\left(\prod_{r=1}^{m}\left[a_{r}, b_{r}\right], L\left(X_{1}, \ldots, X_{m} ; Y\right)\right)$. Então,

$$
\Lambda_{K}\left(x_{1}, \ldots, x_{m}\right)=\int_{a_{m}}^{b_{m}} d_{s_{m}} \cdots \int_{a_{1}}^{b_{1}} d_{s_{1}} K\left(s_{1}, \ldots, s_{m}\right) x_{1}\left(s_{1}\right) \cdots x_{m}\left(s_{m}\right) .
$$

OBservaÇÃo. Para funções contínuas a integral interior de Dushnik e a integral de Riemann-Stieltjes coincidem. Isto é dado pelo teorema 2.2.2 e a coincidência em uma variável (teorema 1.3.1).

\subsection{Teoremas de representação integral}

Apresentamos agora teoremas de representação do tipo Riesz para operadores multilineares definidos sobre espaços de funções regradas.

O conhecido teorema de representação integral de Riesz, para funcionais lineares contínuos sobre $C([a, b], \mathbb{R})$, deu passo a generalizações a dimensões maiores como as de Fréchet (bilineares) e Morse-Transue (multilineares); isto está esquematizado na TABELA 3.

Numa outra direção, o teorema de Riesz, motivou um análogo em $G([a, b], \mathbb{R})$, devido a Kalterborn. Este último foi generalizado para o contexto de espaços de Banach por Hönig e daí surgiram os análogos ao de Fréchet e Morse-Transue (vide TABELA 2).

Os casos em que o espaço de chegada do operador à representar, é um espaço de funções (particularmente o caso dos operadores causais) só tinham sido provados para o caso $m=1$ (vide TABELAS 4 e 5 ). 
DEFINIÇÃo 2.3.1. Seja $K: \prod_{r=1}^{m}\left[a_{r}, b_{r}\right] \longrightarrow L\left(X_{1}, \ldots, X_{m} ; Z\right)$. Dizemos que $K \in S F_{a^{m}}\left(\prod_{r=1}^{m}\left[a_{r}, b_{r}\right], L\left(X_{1}, \ldots, X_{m} ; Z\right)\right)$ se $K\left(s_{1}, \ldots, s_{i-1}, a_{i}, s_{i+1}, \ldots, s_{m}\right)=0$, para todo $i, i=1, \ldots, m$.

OBSERVAÇÃo. $S F_{a^{m}}\left(\prod_{r=1}^{m}\left[a_{r}, b_{r}\right], L\left(X_{1}, \ldots, X_{m} ; Z\right)\right)$ é um espaço vetorial com norma $S F[\cdot]$.

TEOREMA 2.3.1. A aplicação $K \longmapsto \Lambda_{K}$, onde

$$
\Lambda_{K}\left(x_{1}, \ldots, x_{m}\right)=\int_{a_{m}}^{b_{m}} d_{s_{m}} \cdots \int_{a_{1}}^{b_{1}} d_{s_{1}} K\left(s_{1}, \ldots, s_{m}\right) x_{1}\left(s_{1}\right) \cdots x_{m}\left(s_{m}\right),
$$

é uma isometria entre os dois espaços de Banach,

$$
S F_{a^{m}}\left(\prod_{r=1}^{m}\left[a_{r}, b_{r}\right], L\left(X_{1}, \ldots, X_{m} ; Z\right)\right)
$$

$e$

$$
L\left(G^{-}\left(\left[a_{1}, b_{1}\right], X_{1}\right), \ldots, G^{-}\left(\left[a_{m}, b_{m}\right], X_{m}\right) ; Z\right)
$$

Além disso, $K\left(s_{1}, \ldots, s_{m}\right)\left(\bar{x}_{1}, \ldots, \bar{x}_{m}\right)=\Lambda_{K}\left(\chi_{\left(a_{1}, s_{1}\right]} \bar{x}_{1}, \ldots, \chi_{\left(a_{m}, s_{m}\right]} \bar{x}_{m}\right) e$ $\left\|\Lambda_{K}\right\|=S F[K]$.

Prova: Pelo lema 2.2.1, a aplicação está bem definida, é contínua e linear. Da parte (iii) do mesmo lema, temos que $\|\Lambda\| \leq S F\left[K^{\prime}\right]$.

Injetividade. Se $K \neq 0$ existem $\tau_{r} \in\left(a_{r}, b_{r}\right]$ e $\bar{x}_{r} \in X_{r}, r=1, \ldots, m$, tais que

$$
K\left(\tau_{1}, \ldots, \tau_{m}\right)\left(\bar{x}_{1}, \ldots, \bar{x}_{m}\right) \neq 0
$$

Seja $x_{r}=\chi_{\left(a_{r}, \tau_{r}\right]} \bar{x}_{r} \in G^{-}\left(\left[a_{r}, b_{r}\right], X_{r}\right)$, então $\Lambda_{k} \neq 0$, pois

$$
\begin{aligned}
\Lambda_{K} x & =\int_{a_{m}}^{b_{m}} \cdots \int_{a_{1}}^{b_{1}} d_{s_{1} \ldots s_{m}} K\left(s_{1}, \ldots, s_{m}\right)\left(\chi_{\left(a_{1}, \tau_{1}\right]} \bar{x}_{1}, \ldots, \chi_{\left(a_{m}, \tau_{m}\right]} \bar{x}_{m}\right) \\
& =K\left(\tau_{1}, \ldots, \tau_{m}\right)\left(\bar{x}_{1}, \ldots, \bar{x}_{m}\right) .
\end{aligned}
$$


Sobrejetividade. Dada $\Lambda \in L\left(G^{-}\left(\left[a_{1}, b_{1}\right], X_{1}\right), \ldots, G^{-}\left(\left[a_{m}, b_{m}\right], X_{m}\right) ; Z\right)$, se existe $K \in S F_{a^{m}}\left(\prod_{r=1}^{m}\left[a_{r}, b_{r}\right], L\left(X_{1}, \ldots, X_{m} ; Z\right)\right)$ tal que $\Lambda=\Lambda_{K}$, então

$$
K\left(\tau_{1}, \ldots, \tau_{m}\right)\left(\bar{x}_{1}, \ldots, \bar{x}_{m}\right)=\Lambda\left(\chi_{\left(a_{1}, \tau_{1}\right]} \bar{x}_{1}, \ldots, \chi_{\left(a_{m}, \tau_{m}\right]} \bar{x}_{m}\right),
$$

$\tau_{r} \in\left(a_{r}, b_{r}\right]$ e $\bar{x}_{r} \in X_{r}, r=1, \ldots, m$. Tomemos esta, como uma definição para $K$.

Devemos provar que a) $S F[K] \leq\|\Lambda\|$ e b) $\Lambda_{K}=\Lambda$.

$$
\begin{aligned}
a) S F_{P}[K] & =\sup _{\left\|\bar{x}_{i(r)}\right\| \leq 1}\left\{\left\|\sum_{i(1), \ldots, i(m)}^{n(P)} \Delta_{i(1)} \ldots \Delta_{i(m)} K\left(\bar{x}_{i(1)}, \ldots, \bar{x}_{i(m)}\right)\right\|: \bar{x}_{i(r)} \in X_{r}\right\} \\
& =\sup _{\left\|\bar{x}_{i(r)}\right\| \leq 1}\left\{\left\|\sum_{i(1), \ldots, i(m)}^{n(P)} \Lambda\left(\chi_{\left(t_{i(1)-1}, t_{i(1)}\right)} \bar{x}_{i(1)}, \ldots, \chi_{\left(t_{i(m)-1}, t_{i(m)}\right)} \bar{x}_{i(m)}\right)\right\|\right\} \\
& =\sup _{\left\|\bar{x}_{i(r)}\right\| \leq 1}\left\{\left\|\Lambda\left(\sum_{i(1)=1}^{n\left(P_{1}\right)} \chi_{\left(t_{i(1)-1}, t_{i(1)}\right]} \bar{x}_{i(1)}, \ldots, \sum_{i(m)=1}^{n\left(P_{m}\right)} \chi_{\left(t_{i(m)-1}, t_{i(m)}\right]} \bar{x}_{i(m)}\right)\right\|\right\} \\
& \leq\|\Lambda\| .
\end{aligned}
$$

b) Sendo $\Lambda, \Lambda_{K} \in L\left(G^{-}\left(\left[a_{1}, b_{1}\right], X_{1}\right), \ldots, G^{-}\left(\left[a_{m}, b_{m}\right], X_{m}\right) ; Z\right)$, para mostrar a igualdade é suficiente verificar que os operadores coincidem nos elementos da forma

$$
\chi_{\left(a_{1}, r_{1}\right)} \bar{x}_{1}, \ldots, \chi_{\left(a_{m}, \tau_{m}\right)} \bar{x}_{m}
$$

pois estes constituem um conjunto total em

$$
G^{-}\left(\left[a_{1}, b_{1}\right], X_{1}\right), \ldots, G^{-}\left(\left[a_{m}, b_{m}\right], X_{m}\right)
$$

respectivamente. De fato,

$$
\begin{aligned}
\Lambda_{K}\left(\chi_{\left(a_{1}, \tau_{1}\right]}\right. & \left.\bar{x}_{1}, \ldots, \chi_{\left(a_{m}, \tau_{m}\right]} \bar{x}_{m}\right)= \\
& =\int_{a_{m}}^{b_{m}} \ldots \int_{a_{1}}^{b_{m}} d_{s_{1} \ldots s_{m}} K\left(s_{1}, \ldots, s_{m}\right)\left(\chi_{\left\{a_{1}, \tau_{1}\right]}\left(s_{1}\right) \bar{x}_{1}, \ldots, \chi_{\left(a_{m}, \tau_{m}\right)}\left(s_{m}\right) \bar{x}_{m}\right) \\
& =K\left(\tau_{1}, \ldots, \tau_{m}\right)\left(\bar{x}_{1}, \ldots, \bar{x}_{m}\right) \\
& =\Lambda\left(\chi_{\left(a_{1}, \tau_{1}\right)} \bar{x}_{1}, \ldots, \chi_{\left(a_{m}, \tau_{m}\right)} \bar{x}_{m}\right) .
\end{aligned}
$$


Mostramos à seguir um esquema referente aos teoremas de representação no espaço das funções regradas

\begin{tabular}{|c|c|c|}
\hline$m$ & $L\left(G^{-}\left(\prod_{r=1}^{m}\left[a_{r}, b_{r}\right], X\right), Z\right)$ & $L\left(\prod_{r=1}^{m} G^{-}\left(\left[a_{r}, b_{r}\right], X\right), Z\right)$ \\
\hline \hline 1 & \multicolumn{2}{|c|}{ Hönig(1975) [12] } \\
\hline 2 & Prandini(1993) [23] & Prandini(1993) [23] \\
\hline$\vdots$ & $\vdots$ & $\times$ \\
\hline $\mathrm{N}$ & Prandini(1993) [23] & TEOREMA 2.3.1 \\
\hline
\end{tabular}

TABela 2

esta tabela é inspirada de um modo natural nos resultados já conhecidos nas funções contínuas a valores reais:

\begin{tabular}{|c|c|c|}
\hline$m$ & $L\left(C\left(\prod_{r=1}^{m}\left[a_{r}, b_{r}\right], \mathbb{R}\right), \mathbb{I R}\right)$ & $L\left(\prod_{r=1}^{m} C\left(\left[a_{r}, b_{r}\right], \mathbb{R}\right), \mathbb{I R}\right)$ \\
\hline \hline 1 & \multicolumn{2}{|c|}{$\operatorname{Riesz(1914)[24]}$} \\
\hline 2 & Morse-Transue(1950) [19] & Fréchet(1915) [8] \\
\hline$\vdots$ & $\vdots$ & $\times$ \\
\hline $\mathrm{N}$ & Morse-Transue(1950) [19] & Morse-Transue(1950) [18] \\
\hline
\end{tabular}

TABela 3

Consideremos agora o caso em que o espaço de chegada é também um espaço de funções. Precisamos, antes, da seguinte 
DEFINIÇÃO 2.3.2. Seja $K:[a, b] \times \prod_{r=1}^{m}\left[a_{r}, b_{r}\right] \longrightarrow L\left(X_{1}, \ldots, X_{m} ; Y\right)$. Definimos $K^{t}: \prod_{r=1}^{m}\left[a_{r}, b_{r}\right] \longrightarrow L\left(X_{1}, \ldots, X_{m} ; Y\right)$ e $K_{s^{m}}:[a, b] \longrightarrow L\left(X_{1}, \ldots, X_{m} ; Y\right)$ por $K^{t}\left(s_{1}, \ldots, s_{m}\right)=K\left(t, s_{1}, \ldots, s_{m}\right)=K_{s^{m}}(t)$

Além disso, consideramos as propriedades:

$\left(G^{\sigma}\right): K$ é simplesmente regrada como função de $t$, isto é

$$
K_{s^{m}} \in G^{\sigma}\left([a, b], L\left(X_{1}, \ldots, X_{m} ; Y\right)\right) .
$$

$\left(S F^{u}\right): K$ é uniformemente de semivariação de Fréchet limitada como função de $\left(s_{1}, \ldots, s_{m}\right)$, isto é

$$
S F^{u}\left[K^{\prime}\right]=\sup _{t \in[a, b]} S F\left[K^{t}\right]<\infty
$$

$\left(S F_{a^{m}}^{u}\right): K \quad$ satisfaz $\left(S F^{u}\right)$ e $K^{t} \in S F_{a^{m}}\left(\prod_{r=1}^{m}\left[a_{r}, b_{r}\right], L\left(X_{1}, \ldots, X_{m} ; Z\right)\right)$ para todo $t \in[a, b]$.

Se $K$ verifica $\left(G^{\sigma}\right)$ e $\left(S F^{u}\right)$, escrevemos

$$
K \in G^{\sigma} \cdot S F^{u}\left([a, b] \times \prod_{r=1}^{m}\left[a_{r}, b_{r}\right], L\left(X_{1}, \ldots, X_{m} ; Y\right)\right) .
$$

Análogamente define-se $G^{\sigma} \cdot S F_{a^{m}}^{u}$. Em particular, precisamos da definição de $G^{\sigma} . S F^{u}$ para o caso $m=1$.

Teorema 2.3.2. A aplicação $K \longmapsto \Lambda_{K^{*}}$, onde

$$
\Lambda_{K^{-}}\left(x_{1}, \ldots, x_{m}\right)(t)=\int_{a_{m}}^{b_{m}} d_{s_{m}} \cdots \int_{a_{1}}^{b_{1}} d_{s_{1}} K\left(t, s_{1}, \ldots, s_{m}\right) x_{1}\left(s_{1}\right) \cdots x_{m}\left(s_{m}\right)
$$


é uma isometria entre os dois espaços de Banach,

$$
G^{\sigma} \cdot S F_{a^{m}}^{u}\left([a, b] \times \prod_{r=1}^{m}\left[a_{r}, b_{r}\right], L\left(X_{1}, \ldots, X_{m} ; Y\right)\right)
$$

e

$$
L\left(G^{-}\left(\left[a_{1}, b_{1}\right], X_{1}\right), \ldots, G^{-}\left(\left[a_{m}, b_{m}\right], X_{m}\right) ; G([a, b], Y)\right)
$$

$\operatorname{com} K\left(t, s_{1}, \ldots, s_{m}\right)\left(\bar{x}_{1}, \ldots, \bar{x}_{m}\right)=\Lambda_{K}\left(\chi_{\left(a_{1}, s_{1}\right]} \bar{x}_{1}, \ldots, \chi_{\left(a_{m}, s_{m}\right]} \bar{x}_{m}\right)(t) \quad e$

$\left\|\Lambda_{K}\right\|=S F^{u}[K]$

Prova: Para $t \in[a, b], K_{s^{m}}$ é de semivariação de Fréchet limitada. Por outro lado, $x_{r}$ é regrada, $r=1, \ldots, m$. Então $\Lambda_{K}\left(x_{1}, \ldots, x_{m}\right)(t)$ está bem definida. Assim como no teorema anterior, temos que a linearidade e a injetividade são consequências imediatas da definição. Além disso, para $t \in[a, b]$,

$$
\left\|\left(\Lambda_{K} x\right)(t)\right\| \leq S F\left[K^{t}\right]\left\|x_{1}\right\| \cdots\left\|x_{m}\right\|
$$

Logo,

$$
\left\|\Lambda_{K} x\right\| \leq S F^{u}[K]\left\|x_{1}\right\| \cdots\left\|x_{m}\right\|
$$

Portanto,

$$
\left\|\Lambda_{K}\right\| \leq S F^{u}[K]
$$

Sobrejetividade. Seja $\Lambda \in L\left(G^{-}\left(\left[a_{1}, b_{1}\right], X_{1}\right), \ldots, G^{-}\left(\left[a_{m}, b_{m}\right], X_{m}\right) ; G([a, b], Y)\right)$. Pelo teorema anterior, existe $\bar{K} \in S F_{a^{m}}\left(\prod_{r=1}^{m}\left[a_{r}, b_{r}\right], L\left(X_{1}, \ldots, X_{m} ; G([a, b], Y)\right)\right.$ tal que

$$
\Lambda x=\int_{a_{m}}^{b_{m}} d_{s_{m}} \cdots \int_{a_{1}}^{b_{1}} d_{s_{1}} \bar{K}\left(s_{1}, \ldots, s_{m}\right) x_{1}\left(s_{1}\right) \cdots x_{m}\left(s_{m}\right),
$$

onde

$$
\bar{K}\left(s_{1}, \ldots, s_{m}\right)\left(\bar{x}_{1}, \ldots, \bar{x}_{m}\right)=\Lambda\left(\chi_{\left(a_{1}, s_{1}\right]} \bar{x}_{1}, \ldots, \chi_{\left(a_{m}, s_{m}\right]} \bar{x}_{m}\right) .
$$


Definindo

$$
K:[a, b] \times \prod_{r=1}^{m}\left[a_{r}, b_{r}\right] \longrightarrow L\left(X_{1}, \ldots, X_{m} ; Y\right)
$$

por

$$
K\left(t, s_{1}, \ldots, s_{m}\right)\left(\bar{x}_{1}, \ldots, \bar{x}_{m}\right)=\left(\bar{K}^{\prime}\left(s_{1}, \ldots, s_{m}\right)\left(\bar{x}_{1}, \ldots, \bar{x}_{m}\right)\right)(t),
$$

temos,

$$
\Lambda\left(\chi_{\left(a_{1}, s_{1}\right]} \bar{x}_{1}, \ldots, \chi_{\left(a_{m}, s_{m}\right]} \bar{x}_{m}\right)(t)=K\left(t, s_{1}, \ldots, s_{m}\right)\left(\bar{x}_{1}, \ldots, \bar{x}_{m}\right) .
$$

E portanto,

$$
(\Lambda x)(t)=\int_{a_{m}}^{b_{m}} d_{s_{m}} \cdots \int_{a_{1}}^{b_{1}} d_{s_{1}} K\left(t, s_{1}, \ldots, s_{m}\right) x_{1}\left(s_{1}\right) \cdots x_{m}\left(s_{m}\right) .
$$

Mostremos agora que $K \in G^{\sigma} \cdot S F_{a^{m}}^{u}\left([a, b] \times \prod_{r=1}^{m}\left[a_{r}, b_{r}\right], L\left(X_{1}, \ldots, X_{m} ; Y\right)\right)$.

a) $K\left(t, a_{1}, \ldots, a_{m}\right)\left(\bar{x}_{1}, \ldots, \bar{x}_{m}\right)=\left(\bar{K}^{\prime}\left(a_{1}, \ldots, a_{m}\right)\left(\bar{x}_{1}, \ldots, \bar{x}_{m}\right)\right)(t)=0$.

b) $K$ é uniformemente de semivariação de Fréchet em $\left(s_{1}, \ldots, s_{m}\right)$.

De fato, sejam $P=\prod_{r=1}^{m} P_{r}, P_{r} \in \mathbb{P}\left[a_{r}, b_{r}\right]$ e $\bar{x}_{i(r)} \in X_{r} \quad$ com $\left\|\bar{x}_{i(r)}\right\| \leq 1, \quad$ para todo $i(r), 1 \leq i(r) \leq n\left(P_{r}\right)$, e para todo $r=1, \ldots, m$. Então,

$$
\begin{aligned}
\left\|\sum_{i(1), \ldots, i(m)}^{n(P)} \Delta_{i(1)} \ldots \Delta_{i(m)} K(t)\left(\bar{x}_{i(1)}, \ldots, \bar{x}_{i(m)}\right)\right\|= \\
\quad=\left\|\Lambda\left(\sum_{i(1)=1}^{n\left(P_{1}\right)} \chi_{\left(t_{i(1)-1}, t_{i(1)}\right)} \bar{x}_{i(1)}, \ldots \sum_{i(m)=1}^{n\left(P_{m}\right)} \chi_{\left(t_{i(m)-1}, t_{i(m)}\right)} \bar{x}_{i(m)}\right)(t)\right\| \\
\leq\left\|\Lambda\left(\sum_{i(1)=1}^{n\left(P_{1}\right)} \chi_{\left(t_{i(1)-1}, t_{i(1)}\right)} \bar{x}_{i(1)}, \ldots \sum_{i(m)=1}^{n\left(P_{m}\right)} \chi_{\left(t_{i(m)-1}, t_{i(m)}\right)} \bar{x}_{i(m)}\right)\right\| \\
\leq\|\Lambda\|\left\|\sum_{i(1)=1}^{n\left(P_{1}\right)} \chi_{\left(t_{i(1)-1}, t_{i(1)}\right)} \bar{x}_{i(1)}\right\| \ldots \sum_{i(m)=1}^{n\left(P_{m}\right)} \chi_{\left(t_{i(m)-1}, t_{i(m)}\right)} \bar{x}_{i(m)} \| \\
\leq\|\Lambda\| .
\end{aligned}
$$


Logo,

$$
S F\left[K^{r t}\right] \leq\|\Lambda\|, \quad \text { para todo } t \in[a, b] .
$$

Donde,

$$
S F^{u}[K] \leq\|\Lambda\|
$$

c) $K$ é simplesmente regrada como função de $t$, pois como $\Lambda$ toma valores em $G([a, b], Y)$, pela definição de $K$ temos que. para todo $\left(s_{1}, \ldots, s_{m}\right)$ e para todo $\left(\bar{x}_{1}, \ldots, \bar{x}_{m}\right)$, a função

$$
\phi:[a, b] \longrightarrow Y
$$

definida por

$$
\phi(t)=K\left(t, s_{1}, \ldots, s_{m}\right)\left(\bar{x}_{1}, \ldots, \bar{x}_{m}\right)
$$

é regrada.

Com este resultado conseguimos completar a tabela de teoremas de representação, quando o espaço de chegada é um espaço de funções regradas.

\begin{tabular}{|c|c|}
\hline$m$ & $\left.L\left(\prod_{r=1}^{m} G^{-}\left(\left[a_{r}, b_{r}\right], X\right), G^{-}([a, b], Y)\right)\right)$ \\
\hline \hline 1 & $\operatorname{Arbex}(1976)[1]$ \\
\hline 2 & $\times$ \\
\hline$\vdots$ & $\vdots$ \\
\hline $\mathrm{N}$ & TEOREMA 2.3 .2 \\
\hline
\end{tabular}

TABELA 4 
Para finalizar esta seção, tratamos o caso dos operadores causais.

DEFINIÇÃO 2.3.3. Seja $K^{\prime} \in G^{\sigma} \cdot S F^{u}\left([a, b]^{m+1}, L_{m}(X ; Y)\right)$ onde $[a, b]^{m+1}=\prod_{r=1}^{m+i}[a, b]$ e seja $L_{m}(X ; Y)=L\left(X^{m} ; Y\right)$ com $X^{m}=\prod_{r=1}^{m} X$. Se, para todo $x \in X$, a função $K_{\Delta}:[a, b] \longrightarrow Y$ definida por $t \longmapsto K(t, \ldots, t)(x, \ldots, x)$ é regrada, dizemos que $K$ é simplesmente regrada na diagonal e escrevemos

$$
K \in G_{\Delta}^{\sigma} \cdot S F^{u}\left([a, b]^{m+1}, L_{m}(X ; Y)\right)
$$

Se além disso $K_{\Delta}(t)=0$, para todo $t \in[a, b]$, então dizemos que $K$ se anula na diagonal e escrevemos

$$
K \in G_{0}^{\sigma} \cdot S F^{u}\left([a, b]^{m+1}, L_{m}(X ; Y)\right)
$$

Do modo análogo define-se $G_{0}^{\sigma} \cdot S V^{u}\left([a, b]^{2}, L(X ; Y)\right)$.

DEFINIÇÃo 2.3.4. $P \in L_{m}(G([a, b], X) ; G([a, b], Y))$ é um operador causal se para todo $x \in G([a, b], X)$ e para todo $T \in[a, b]$,

$$
x_{\left.\right|_{[a, T]}}=0 \Longrightarrow P(x, \ldots, x)_{\left.\right|_{[a, T]}}=0 .
$$

DEFINIÇÃo 2.3.5. Seja $K \in G^{\sigma} \cdot S F^{u}\left([a, b]^{m+1}, L_{m}(X ; Y)\right)$. Dado $x=\left(x_{1}, \ldots, x_{m}\right)$ com $x_{r} \in G^{-}([a, b], X), r=1, \ldots, m$, definimos

$$
(k x)(t)=\int_{a}^{t} d_{s_{m}} \cdots \int_{a}^{t} d_{s_{1}} K\left(t, s_{1}, \ldots, s_{m}\right) x_{1}\left(s_{1}\right) \cdots x_{m}\left(s_{m}\right),
$$

para $t \in[a, b]$.

À seguir mostramos que os operadores multilineares causais também podem ser representados. 
TeOrema 2.3.3. A aplicação $K \longmapsto k$ é uma isometria entre o espaço de Banach

$$
G_{0}^{\sigma} \cdot S F^{u}\left([a, b]^{m+1}, L_{m}(X ; Y)\right)
$$

e o subespaço dos operadores causais de

$$
L_{m}\left(G^{-}([a, b], X) ; G([a, b], Y)\right)
$$

Além disso, $K\left(t, s_{1}, \ldots, s_{m}\right)\left(\bar{x}_{1}, \ldots, \bar{x}_{m}\right)=k\left(\chi_{\left(a_{1}, t\right]} \bar{x}_{1}, \ldots, \chi_{\left(a_{m}, t\right]} \bar{x}_{m}\right)(t)$ e $\|k\|=S F^{u}[K]$.

Prova: Dada $K \in G^{\sigma} \cdot S F^{u}\left([a, b]^{m+1}, L_{m}(X ; Y)\right)$, definimos

$$
\tilde{K}\left(t, s_{1}, \ldots, s_{m}\right)= \begin{cases}K\left(t, s_{1}, \ldots, s_{m}\right) & \text { se } t \geq s_{r}, \text { para todo } r, r=1, \ldots, m \\ K(t, t, \ldots, t) & \text { se } t<s_{r}, \text { para algum } r, r=1, \ldots, m\end{cases}
$$

Se $K \in G_{\Delta}^{\sigma} \cdot S F^{u}\left([a, b]^{m+1}, L_{m}(X ; Y)\right)$, então $\tilde{K}^{\tau} \in G_{\Delta}^{\sigma} \cdot S F^{u}\left([a, b]^{m+1}, L_{m}(X ; Y)\right)$. Além disso, temos

$$
\begin{aligned}
(k x)(t) & =\int_{a}^{t} d_{s_{m}} \cdots \int_{a}^{t} d_{s_{1}} K\left(t, s_{1}, \ldots, s_{m}\right) x_{1}\left(s_{1}\right) \cdots x_{m}\left(s_{m}\right) \\
& =\int_{a}^{b} d_{s_{m}} \cdots \int_{a}^{b} d_{s_{1}} \tilde{K}\left(t, s_{1}, \ldots, s_{m}\right) x_{1}\left(s_{1}\right) \cdots x_{m}\left(s_{m}\right) .
\end{aligned}
$$

Assim, pelo teorema anterior, temos que $k x \in G([a, b], Y)$, para todo $x=\left(x_{1}, . ., x_{m}\right)$ com $x_{r} \in G([a, b], X), r=1, \ldots, m$.

Reciprocamente, se $\Lambda \in L_{m}\left(G^{-}([a, b], X) ; G([a, b], Y)\right)$ é um operador causal, então, também pelo teorema anterior, existe $\bar{K}^{\sigma} \in G^{\sigma} \cdot S F_{a^{u}}^{u}\left([a, b]^{m+1}, L_{m}(X ; Y)\right)$ tal que

$$
(\Lambda x)(t)=\int_{a}^{b} d_{s_{m}} \cdots \int_{a}^{b} d_{s_{1}} \bar{K}\left(t, s_{1}, \ldots, s_{m}\right) x_{1}\left(s_{1}\right) \cdots x_{m}\left(s_{m}\right),
$$

onde $\bar{K}\left(t, s_{1}, \ldots, s_{m}\right)^{\left(s_{1}, \bar{x}_{m}\right)}=\Lambda\left(\chi_{\left(a_{1}, s_{1}\right]} \bar{x}_{1}, \ldots, \chi_{\left(a_{m}, s_{m}\right]} \bar{x}_{m}\right)(t), t \in[a, b], \bar{x}_{r} \in X \quad \mathrm{e}$ $s_{r} \in(a, b], r=1, \ldots, m$. 
Como $\Lambda$ é causal, para $s_{r} \geq t, r=1, \ldots, m$, temos

$$
\Lambda\left(\chi_{\left(a, s_{1}\right]} \bar{x}_{1}, \ldots, \chi_{(a, s m]} \bar{x}_{m}\right)(t)=\Lambda\left(\chi_{(a, t]} \bar{x}_{1}, \ldots, \chi_{(a, t]} \bar{x}_{m}\right)(t),
$$

isto é,

$$
\bar{K}\left(t, s_{1}, \ldots, s_{m}\right)\left(\bar{x}_{1}, \ldots, \bar{x}_{m}\right)=\bar{K}(t, \ldots, t)\left(\bar{x}_{1}, \ldots, \bar{x}_{m}\right) .
$$

Assim,

$$
(\Lambda x)(t)=\int_{a}^{t} d_{s_{m}} \cdots \int_{a}^{t} d_{s_{1}} \bar{K}\left(t, s_{1}, \ldots, s_{m}\right) x_{1}\left(s_{1}\right) \cdots x_{m}\left(s_{m}\right) .
$$

Fazendo $x_{r}\left(s_{r}\right)=\bar{c}_{r}$, resulta que

$$
\begin{aligned}
(\Lambda x)(t) & =\int_{a}^{t} d_{s_{m}} \cdots \int_{a}^{t} d_{s_{1}} \bar{K}\left(t, s_{1}, \ldots, s_{m}\right) \bar{c}_{1} \cdots \bar{c}_{m} \\
& =\bar{K}(t, \ldots, t)+\sum_{s_{i} \in\{t, a\}}(-1)^{\#(a)} \bar{K}\left(t, s_{1}, \ldots, s_{m}\right) \bar{c}_{1} \cdots \bar{c}_{m}
\end{aligned}
$$

$\operatorname{com} \#(a)=\sum_{i=1}^{m} \delta_{i}$, onde $\delta_{i}=\left\{\begin{array}{ccc}1, & \text { se } & s_{i}=a \\ 0, & \text { se } & s_{i}=t .\end{array}\right.$

Como $\Lambda x$ é simplesmente regrada e $K \in G^{\sigma} \cdot S F^{u}$, então a aplicação

$$
t \longmapsto \bar{K}(t, s, \ldots, s)
$$

é regrada. E portanto,

$$
t \longmapsto \bar{K}(t, \ldots, t)
$$

é regrada. Donde,

$$
\bar{K} \in G_{\Delta}^{\sigma} \cdot S F^{u}\left([a, b]^{m+1}, L_{m}(X ; Y)\right)
$$

Definindo $K\left(t, s_{1}, \ldots, s_{m}\right)=\bar{K}\left(t, s_{1}, \ldots, s_{m}\right)-\bar{K}(t, t, \ldots, t)$, temos que $K \in G_{\Delta}^{\sigma} \cdot S F^{u}$ e $K(t, \ldots, t)=0$, para todo $t \in[a, b]$. Assim,

$$
K_{\Delta}(t)=0, \text { para todo } t \in[a, b]
$$


De $(\Delta)$ e $(0)$, temos que $K \in G_{0}^{\sigma} \cdot S F^{n}$ ?

Além disso, com tal $K$ definido, resulta que

$$
(\Lambda x)(t)=\int_{a}^{t} d_{s_{m}} \cdots \int_{a}^{t} d_{s_{1}} K\left(t, s_{1}, \ldots, s_{m}\right) x_{1}\left(s_{1}\right) \cdots x_{m}\left(s_{m}\right)=(k x)(t)
$$

Então, $\Lambda x=k x$. E portanto, $\Lambda=k$.

Fechamos assim a coleção de teoremas de representação, completando a última tabela:

\begin{tabular}{|c|c|}
\hline$m$ & Causais $\left.\subset L\left(\prod_{r=1}^{m} G^{-}\left(\left[a_{r}, b_{r}\right], X\right), G^{-}([a, b], Y)\right)\right)$ \\
\hline \hline 1 & Hönig $(1979)[13]$ \\
\hline 2 & $\times$ \\
\hline$\vdots$ & $\vdots$ \\
\hline $\mathrm{N}$ & TeOrema 2.3 .3 \\
\hline
\end{tabular}

TABELA 5

\subsection{Aproximação de operadores não lineares}

A aproximação de operadores não lineares por operadores polinomiais foi concretizada em 1980 por Istrăţescu [16], mostrando que todo operador contínuo, sobre um subconjunto compacto de um espaço de Banach, pode ser aproximado uniformemente por operadores polinomiais (Teorema de Weierstrass para espaços de Banach). Mais tarde, Daugavet-Baesler [3], trataram de um problema similar, onde o espaço de Ba- 
nach é um espaço de funções $\left(C[a, b]\right.$ ou $\left.L_{p}[a, b]\right)$ e os operadores polinomiais são somas parciais de séries de Volterra-Wiener.

Aqui trabalhamos no contexto das funções regradas. Veremos que os operadores polinomiais também têm forma de séries de Volterra-Wiener truncadas, embora as integrais correspondentes sejam do tipo Stieltjes. Para tal, foi necessária a teoria de representação integral para operadores m-lineares feita na seção 2.3 , que nos permite expressar os polinomiais do teorema de Weierstrass por operadores polinomiais de Volterra-Stieltjes.

DEFINIÇÃo 2.4.1. Seja $A \subset X$ um compacto não vazio e consideremos os espaços de operadores:

- $C(A, X)=\{\Lambda: A \longrightarrow X$ tal que $\Lambda$ é contínuo $\}$

- $P(A, X)=\{P: A \longrightarrow X$ tal que $P$ é um polinomial contínuo $\}$

Teorema 2.4.1. [16, Theorem 12.5.23] (Teorema de Weierstrass) $P(A, X)$ é denso em $C(A, X)$.

OBServação. Com relação à prova do Teorema de Weierstrass para espaços de Banach, queremos destacar que foram utilizados três importantes teoremas da Análise Funcional: o Teorema de Hahn-Banach, o Teorema de Krein-Milman e o Teorema de Riesz-Katutani.

DEFINIÇÃo 2.4.2. Se $h_{m} \in G^{\sigma} \cdot S F_{a m}^{u}\left([a, b]^{m+1}, L_{m}(X ; X)\right)$, o operador

$$
P_{m}: G^{-}([a, b], X) \longrightarrow G^{-}([a, b], X),
$$


definido por

$$
\left(P_{m} x\right)(t)=h_{0}(t)+\sum_{n=1}^{m} \int_{a}^{b} d_{s_{n}} \cdots \int_{a}^{b} d_{s_{1}} h_{n}\left(t, s_{1}, \ldots, s_{n}\right) x\left(s_{1}\right) \cdots x\left(s_{n}\right),
$$

é chamado de Polinomial de Volterra-Stieltjes de grau $m$.

Teorema 2.4.2. Sejam $A \subset G^{-}([a, b], X)$ compacto e $P: A \rightarrow G^{-}([a, b], X)$ contínuo. Então para todo $\epsilon>0$, existe um polinomial de Volterra-Stieltjes $P_{m}$ tal que

$$
\left\|P x-P_{m} x\right\|<\epsilon,
$$

para todo $x \in A$.

Prova: Do teorema 2.4.1 tem-se que todo operador contínuo, definido sobre um subconjunto compacto de um espaço de Banach, pode ser aproximado uniformemente por polinomiais. Pelo teorema 2.3.2 estes são operadores de Volterra-Stieltjes. 


\section{Capítulo 3}

\section{Expansões para sistemas não}

\section{lineares}

Em 1985, Hönig [15] mostrou a existência, unicidade e dependência contínua dos valores iniciais, no âmbito das funções regradas com valores num espaço de Banach, para a equação integral não linear:

$$
x(t)+\int_{a}^{t} d_{s} K(t, s) f(s, x(s))=u(t), \quad t \in[a, b] .
$$

Devemos destacar que no caso linear em $(K)$, isto é quando $f(s, x(s))=x(t)$, Arbex [1] mostrou que a solução é dada por uma série de Neumann. Além disso a equação linear tem sido bastante estudada, comprovando-se sua qualidade unificadora, pois engloba sistemas extensos tais como: EDO, EDP, Equações do tipo Neutro, com ação impulsiva e de Volterra-Stieltjes (vide [14]).

Usando o resultado de Arbex (sobre séries de Neumann) e os teoremas de repre- 
sentação integral do capítulo 2, conseguimos expressar as soluções do sistema $(K)$ em forma de séries de potência com termos integrais.

\subsection{Representação em séries de Volterra}

As séries de Volterra foram criadas por ele, no final do século passado, como uma generalização das expansões em série de Taylor de uma função. No começo deste século, aplicou-as no estudo de certas equaçòes integrais e íntegro-diferenciais. Mais tarde, Wiener fez aplicações na Teoria de Sistemas. Para uma excelente bibliografia com perspectiva histórica ver o livro de Schetzen [25].

Queremos destacar que as séries de Volterra são uma ferramenta muito utilizada na teoria de sistemas não lineares na engenharia. Mas o fato de não se ter um teorema de representação integral satisfatório em $L_{2}[a, b]$ obriga o uso da teoria de distribuções na justificativa teórica das séries de Volterra. No nosso caso, em que consideramos $G([a, b], X)$, a existência destes teoremas de representação integral fizeram de tais séries uma aplicação dos teoremas de Weierstrass e Taylor.

Consideremos $V \subset G^{-}([a, b], X)$, aberto não vazio.

DEFINIÇÃo 3.1.1. Uma aplicação $T: G^{-}([a, b], X) \longrightarrow G^{-}([a, b], X)$ tem uma expansão de Volterra-Stieltjes de grau $m$, numa vizinhança de $v_{0}$, se existem núcleos $h_{1}, \ldots, h_{m}$ tais que $T\left(v+v_{0}\right)-T\left(v_{0}\right)=\sum_{n=1}^{m} \int_{a}^{t} d_{s_{n}} \cdots \int_{a}^{t} d_{s_{1}} h_{n}\left(t, s_{1}, \ldots, s_{n}\right) v\left(s_{1}\right) \cdots v\left(s_{n}\right)+R_{m}\left(v_{0}, v\right)$, 
onde,

$$
\lim _{\lambda \rightarrow 0} \frac{\left\|R_{m}\left(v_{0}, \lambda v\right)\right\|}{\lambda^{m}}=0 .
$$

Se existem núcleos $h_{m}, m$ inteiros, tais que

$$
T\left(v+v_{0}\right)-T\left(v_{0}\right)=\sum_{m=1}^{\infty} \int_{a}^{t} d_{s_{m}} \cdots \int_{a}^{t} d_{s_{1}} h_{m}\left(t, s_{1}, \ldots, s_{m}\right) v\left(s_{1}\right) \cdots v\left(s_{m}\right),
$$

com tal série convergindo uniformemente para todo $\|v\|<\delta$, dizemos que $T$ tem representação em séries de Volterra-Stieltjes.

NotaÇÃo. $\delta T\left(v_{0}\right)(v)$ denota a derivada de Gâteaux de $T$ em $v_{0}$ com incremento (na direção de) v. Esta é dada por:

$$
\delta T\left(v_{0}\right)(v)=\lim _{\lambda \rightarrow 0} \frac{T\left(v_{0}+\lambda v\right)-T\left(v_{0}\right)}{\lambda} .
$$

Indutivamente, a derivada de Gâteaux m-ésima de $T$ em $v_{0}$ com incrementos $v_{1}, \ldots, v_{m}$, é dada por:

$\delta^{(m)} T\left(v_{0}\right)\left(v_{1}, \ldots, v_{m}\right)=\lim _{\lambda \rightarrow 0} \frac{\delta^{(m-1)} T\left(v_{0}+\lambda v_{m}\right)\left(v_{1}, \ldots, v_{m-1}\right)-\delta^{(m-1)} T\left(v_{0}\right)\left(v_{1}, \ldots, v_{m-1}\right)}{\lambda}$.

Por último,

$$
\delta^{(m)} T\left(v_{0}\right)[v]=\delta^{(m)} T\left(v_{0}\right)(v, \ldots, v) .
$$

Teorema 3.1.1. [10, Theorem 26.3.5] Seja $T: V \stackrel{C}{\longrightarrow} X \in X$ aberto. Se $T e ́$ $m$-vezes Gâteaux diferenciável em $V$, então $\delta^{(m)} T\left(v_{0}\right)\left(v_{1}, \ldots, v_{m}\right)$ é uma aplicação $m$ linear simétrica em $v_{1}, \ldots, v_{m}$. Além disso, $\delta^{(m)} T\left(v_{0}\right)[v]$ é um polinomial homogêneo de grau $m$ em $v$.

Teorema 3.1.2. [21, Remark of the Theorem 6.1] (TeOrema DE Taylor) Seja $T: V \longrightarrow X$ m-vezes Gâteaux diferenciável em um ponto $v_{0} \in V$. Então,

$$
T\left(v+v_{0}\right)-T\left(v_{0}\right)=\sum_{n=1}^{m} \frac{1}{n !} \delta^{(n)} T\left(v_{0}\right)[v]+R_{m}\left(v_{0}, v\right),
$$


onde,

$$
\lim _{\lambda \rightarrow 0} \frac{\left\|R_{m}\left(v_{0}, \lambda v\right)\right\|}{\lambda^{m}}=0
$$

Teorema 3.1.3. Se $T: V \longrightarrow G^{-}([a, b], X)$ é $m$ vezes Gâteaux diferenciável em $v_{0}$ com $\delta^{(m)} T\left(v_{0}\right)$ causal. Então, $T$ tem uma expansão de Volterra-Stieltjes de grau $m$.

Prova: Pelo Teorema de Taylor, temos que

$$
T\left(v+v_{0}\right)-T\left(v_{0}\right)=\sum_{n=1}^{m} \frac{1}{n !} \delta^{(n)} T\left(v_{0}\right)[v]+R_{m}\left(v_{0}, v\right),
$$

onde,

$$
\lim _{\lambda \rightarrow 0} \frac{\left\|R\left(v_{0}, \lambda v\right)\right\|}{\lambda^{m}}=0 .
$$

Mas, pelo teorema $1.3 .1, \delta^{(m)} T\left(v_{0}\right)$ é uma aplicação $m$-linear, para todo $m$. Além disso, por hipótese, temos que $\delta^{(m)} T$ é causal. Logo, pelo teorema de representação integral (teorema 2.3.3), temos que existe

$$
h_{n} \in G_{0}^{\sigma} \cdot S F^{u}\left([a, b]^{n+1}, L_{n}(X ; Y)\right),
$$

tal que

$$
\delta^{(n)} T\left(v_{0}\right)[v](t)=\int_{a}^{t} d_{s_{n}} \cdots \int_{a}^{t} d_{s_{1}} h_{n}\left(t, s_{1}, \ldots, s_{n}\right) v\left(s_{1}\right) \cdots v\left(s_{n}\right)
$$

com

$$
h_{n}\left(t, s_{1}, \ldots, s_{n}\right)\left(\bar{v}_{1}, \ldots, \bar{v}_{n}\right)=\delta^{(n)} T\left(v_{0}\right)\left(\chi_{\left(a_{1}, t\right]} \bar{v}_{1}, \ldots, \chi_{\left(a_{n}, t\right]} \bar{v}_{n}\right)(t) .
$$

Portanto,

$T\left(v+v_{0}\right)-T\left(v_{0}\right)=\sum_{n=1}^{m} \frac{1}{n !} \int_{a}^{t} d_{s_{n}} \cdots \int_{a}^{t} d_{s_{1}} h_{n}\left(t, s_{1}, \ldots, s_{n}\right) v\left(s_{1}\right) \cdots v\left(s_{n}\right)+R\left(v_{0}, \lambda v\right)$.

Consideremos agora $X$ um espaço de Banach complexo e $V \subset X$ um aberto não vazio. 
DEFINIÇÃo 3.1.2. A aplicação $T: V \longrightarrow Y$ é analítica em $V$ se, e só se,

$$
T(x)=\sum_{n=1}^{\infty} a_{n}[x]^{n}, x \in V
$$

$a_{n}$ polinomial homogêneo de grau $n$ e com a série convergindo absolutamente em $V$ $e$ uniformemente sobre subconjuntos fechados e limitados de $V$.

DEFINIÇÃo 3.1.3. A aplicação $T: V \longrightarrow Y$ é limitada se $T$ aplica conjuntos limitados em conjuntos limitados.

DEFInIÇÃo 3.1.4. A aplicação $T: V \longrightarrow Y$ é localmente limitada se para cada $v_{0} \in V$ existem $B_{R}\left(v_{0}\right)$ e $M>0$ com $\|T(v)\|<M$ para todo $v \in B_{R}\left(v_{0}\right)$.

Teorema 3.1.4. [10, Theorem 3.17.1] Se a aplicação $T: V \longrightarrow Y$ é localmente limitada e diferenciável Gâteaux, então $T$ é analítica em $V$.

Teorema 3.1.5. Seja $T: V \longrightarrow G^{-}([a, b], X)$ um operador analítico em $V$ com $\delta^{(n)}$ causal, então $T$ tem representação em séries de Volterra-Stieltjes.

Prova: Sendo T analítico, existe $R>0$ tal que

$$
T\left(v+v_{0}\right)-T\left(v_{0}\right)=\sum_{n=1}^{\infty} \frac{1}{n !} \delta^{(n)} T\left(v_{0}\right)[v],
$$

uniformemente em $B_{R}\left(v_{0}\right)$.

Como no teorema 3.1.3. o restante é consequência do teorema 1.3.1. e do teorema de representação integral.

Para nossos propósitos necessitamos inverter a série obtida no teorema anterior. Em geral, temos o seguinte teorema de inversão para operadores analíticos em espaços de Banach. 
Doravante consideremos $V \subset X$, um aberto não vazio.

TeOrema 3.1.6. [20] Seja $T: V \longrightarrow X$ um operador localmente limitado. Se $T e ́$ Gâteaux diferenciável com $\delta T\left(v_{0}\right)$ invertível numa vizinhança de $v_{0}$, então existem vizinhanças de $v_{0}$ e $T\left(v_{0}\right)$ onde $T$ tem uma única inversa dada por

$$
T^{-1} u=\sum_{m=1}^{\infty} Q_{m}[u],
$$

com

$$
\begin{gathered}
Q_{1}[u]=\left[\delta T\left(v_{0}\right)\right]^{-1}[u], e \\
Q_{m}[u]=-\left[\delta T\left(v_{0}\right)\right]^{-1} \sum_{n=1}^{m} \sum_{j_{1}+\cdots+j_{n}=m} \frac{1}{n !} \delta^{(m)} T\left(v_{0}\right)\left(Q_{j_{1}}, \ldots, Q_{j_{n}}\right)[u] .
\end{gathered}
$$

\subsection{O operador de Nemytskij}

Na presente seção consideramos o operador não linear

$$
(F x)(s)=f(s, x(s))
$$

onde $f$ é uma aplicação $f:[a, b] \times X \longrightarrow X . F$ é chamado de operador de Nemytskij $\underline{\text { gerado por } f \text { (também: operador de superposição, operador composição ou operador }}$ substituição).

Queremos trabalhar com o operador de Nemytskij definido em $G^{-}([a, b], X)$. Além disso, também aplicando este espaço em sí mesmo. Para isto último, consideremos o espaço

$$
G^{-} \cdot \operatorname{Lip}([a, b] \times X, X)
$$

das funções que são regradas pela esquerda na primeira variável e Lipchitz na segunda, isto é, as $f$ tais que: 
- $t \longmapsto f(t, \bar{x})$ é regrada, para todo $\bar{x} \in X$, e

- existe $L>0$ com

$$
\left\|f\left(t, \bar{x}_{2}\right)-f\left(t, \bar{x}_{1}\right)\right\| \leq \mathrm{L}\left\|\bar{x}_{2}-\bar{x}_{1}\right\|
$$

para todo $t \in[a, b]$.

OBservaÇÃo. $G^{-} \cdot \operatorname{Lip}([a, b] \times X, X)$ é um espaço de Banach quando munido da norma

$$
\|f\|_{\text {Lip }}=\sup \left\{\left\|f_{0}\right\|,[f]\right\}
$$

onde,

$$
\begin{aligned}
f_{0}:[a, b] & \longrightarrow X \\
t & \longmapsto f(t, 0),
\end{aligned}
$$

$e$

$$
[f]=\inf \{\mathrm{L}: \mathrm{L} \text { verifica a desigualdade }(\mathrm{L})\} \text {. }
$$

Teorema 3.2.1. Seja $f:[a, b] \times X \longrightarrow X$ Lipchitz na segunda variável. Então, o operador de Nemytskij $F$, gerado por $f$ aplica $G^{-}([a, b], X)$ em símesmo se, e só se, $f \in G^{-} \cdot \operatorname{Lip}([a, b] \times X, X)$. Além disso, $F$ é limitado.

Prova: Se $F$ aplica $G^{-}([a, b], X)$ em sí mesmo, então é imediato que $f$ é regrada pela esquerda na primeira variável.

Reciprocamente, temos que $(i)(F x)(a)=f(a, x(a))=0$.

Além disso, para todo $t \in[a, b]$, verifica-se

(ii) $(F x)(t-)-(F x)(t)=0$. 
De fato,

$$
\begin{aligned}
\lim _{\tau \uparrow t}\|(F x)(\tau)-(F x)(t)\| & =\lim _{\tau \uparrow t}\|f(\tau, x(\tau))-f(t, x(t))\| \\
& =\lim _{\tau \uparrow t}\|f(\tau, x(\tau))-f(\tau, x(t))+f(\tau, x(t))-f(t, x(t))\| \\
& \leq \operatorname{L} \lim _{\tau \uparrow t}\|x(\tau)-x(t)\|+\lim _{\tau \uparrow t}\|f(\tau, x(t))-f(t, x(t))\| \\
& =0+0=0 .
\end{aligned}
$$

Vejamos agora que $F$ é limitado. Seja $A \subset G^{-}([a, b], X)$ limitado, então existe $r>0$ tal que $x \in B_{r}$ para todo $x \in A$. Assim,

$$
\begin{aligned}
\|F x\|-\|F 0\| & \leq\|F x-F 0\| \\
& =\sup _{t \in[a, b]}\|(F x)(t)-(F 0)(t)\| \\
& =\sup _{t \in[a, b]}\|f(t, x(t))-f(t, 0)\| \\
& \leq \mathrm{L} \sup _{t \in[a, b]}\|x(t)\| \\
& \leq \mathrm{L} r .
\end{aligned}
$$

Logo,

$$
\|F x\| \leq \mathrm{L} r+\left\|f_{0}\right\|
$$

\subsection{O sistema não linear $(K)$}

Estudaremos agora, o sistema não linear dado pela equação

$$
x(t)+\int_{a}^{t} d_{s} K(t, s) f(s, x(s))=u(t), \quad t \in[a, b],
$$


onde tanto as entradas $u$ como as saídas $x$ são funções regradas. O núcleo $K^{\prime}:[a, b] \times[a, b] \longrightarrow L(X)$ e a não linearidade $f:[a, b] \times X \longrightarrow X$ satisfazem condições para a existência e unicidade de soluções de $(K)$.

\subsubsection{Existência de soluções}

Teorema 3.3.1. [15] Sejam $K$ e $f$ tais que $K \in G_{0}^{\sigma} \cdot S V^{u}([a, b] \times[a, b], L(X))$, $f \in G^{-} \cdot \operatorname{Lip}([a, b] \times X, X)$ e $c(K, P)[f]<1$, para alguma $P \in \mathbb{P}[a, b]$. Então, para cada $u \in G([a, b], X)$ existe uma única $x$ que é solução de $(K)$. Além disso a solução depende continuamente de $u, K$ e $f$.

NotaÇÃo. Se $P: a=t_{0}<\cdots<t_{n}=b$, então

$$
c(K, P)=\sup \left\{\left\|K\left(t_{i} \dot{+}, t_{i}\right)\right\| ; \sup _{t_{i} \leq t \leq t_{i+1}} S V_{\left(t_{i}, t\right]}\left[K^{t}\right]: 0 \leq i \leq n-1\right\}
$$

Quando $f(t, x(t))=x(t)$, i.e. no caso linear, Arbex [1] mostrou que o resolvente da solução pode ser expressado por uma série de Neumann:

Teorema 3.3.2. [1, Teorema 3.22] Seja $K \in G_{0}^{\sigma} \cdot S^{\prime} V^{u}([a, b] \times[a, b], L(X))$ tal que $c(K, P)<1$, para alguma $P \in \mathbb{P}[a, b]$. Então, para cada $u \in G([a, b], X)$ existe uma única $x$ que é solução de $(K) e$

$$
x(t)=u(t)-\int_{a}^{t} d_{s} R(t, s) u(s),
$$

com

$$
R=I+\sum_{n=1}^{\infty}(-1)^{n} K^{(n)}, \quad K^{(1)}=K \quad e \quad K^{(m+1)}(t, s)=\int_{s}^{t} d_{\sigma} K^{\prime}(t, s) K^{(m)}(\sigma, s) .
$$




\subsubsection{Exemplo: um circuito não linear}

Consideremos o circuito elétrico não linear:

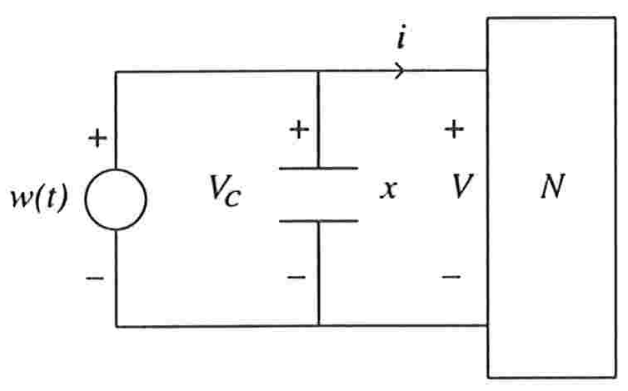

onde $N$ é uma caixa preta tempo-invariante conectada com um capacitor não linear tempo-variante e a conexão paralela é alimentada por uma fonte de corrente $w(t)$. Seja $g$ a resposta de $N$ a um impulso unitário de voltagem. Assim $N$ é caraterizado por

$$
i(t)=\int_{0}^{t} g(t-\tau) V(\tau) d \tau, \quad t \in[0, T]
$$

A característica do capacitor não linear tempo-variante é $V_{c}(t)=f(t, x(t))$, onde $x$ é a carga e $V_{c}$ é a voltagem através das placas. Pela Lei de Kirchoff $V=V_{c}$ e

$$
\dot{x}(t)=w(t)-\int_{0}^{t} g(t-\tau) f(\tau, x(\tau)) d \tau
$$

onde $w$ é a corrente liberada pela fonte.

Consideremos as condições de normalização: $x(0)=0$ e $g(0)=0$.

Integrando em ambas as partes, temos:

$$
x(t)=\int_{0}^{t}\left[w(s)-\int_{0}^{s} g(s-\tau) f(\tau, x(\tau)) d \tau\right] d s .
$$


Se definimos $u(t)=\int_{0}^{t} w(s) d s$, resulta que

$$
x(t)=u(t)-\int_{0}^{t} \int_{0}^{s} g(s-\tau) f(\tau, x(\tau)) d \tau d s .
$$

Fazendo integração por partes, obtemos

$$
\begin{aligned}
x(t) & =u(t)-\left[s \int_{0}^{s} g(s-\tau) f(\tau, x(\tau)) d \tau\right]_{0}^{t}+\int_{0}^{t}\left[s \frac{\partial}{\partial s} \int_{0}^{s} g(s-\tau) f(\tau, x(\tau)) d \tau\right] d s \\
& =u(t)-t \int_{0}^{t} g(t-\tau) f(\tau, x(\tau)) d \tau+\int_{0}^{t} \int_{0}^{s} s g^{\prime}(s-\tau) f(\tau, x(\tau)) d s d \tau .
\end{aligned}
$$

Mudando a ordem de integração, obtemos

$$
\begin{aligned}
x(t) & =u(t)-t \int_{0}^{t} g(t-\tau) f(\tau, x(\tau)) d \tau+\int_{0}^{t} s \int_{0}^{s} g^{\prime}(s-\tau) f(\tau, x(\tau)) d \tau d s . \\
& =u(t)-\int_{0}^{t}\left[t g(t-\tau)-\int_{\tau}^{t} s g^{\prime}(s-\tau) d s\right] f(\tau, x(\tau)) d \tau .
\end{aligned}
$$

Definindo $B(t, \tau)=\operatorname{tg}(t-\tau)-\int_{\tau}^{t} s g^{\prime}(s-\tau) d s \quad$ e $\quad K(t, \sigma)=\int_{0}^{\sigma} B(t, \tau) d \tau$ chegamos à

$$
x(t)=u(t)-\int_{0}^{t} d_{s} K(t, s) f(s, x(s)) .
$$

Observe-se que $(K)$ pode ser expressa por

$$
(I+k F) x=u
$$

com $k$ o operador integral linear já definido (definição 2.3.5. para $m=1$ ), $F$ o operador de Nemytskij e $I$ a identidade em $G^{-}([a, b], X)$.

Fazendo $T=I+k F$, temos então que $(k)$ fica da forma:

$$
T x=u
$$


O teorema 3.1.6. nos dá uma maneira de expressar a solução de $(T)$ em forma de série de potências. Para alcançar este propósito precisamos das seguintes hipóteses:

(Hip.1) As condições de existência e unicidade dadas no teorema 3.3.1.

(Hip.2) Existe $\delta f\left(t, x_{0}\right)[\cdot]$ em uma vizinhança de $x_{0}$.

(Hip.3) $\|\delta F\| \leq \inf \left\{1, \frac{1}{S V[K]}\right\}$.

(Hip.4) Se existe $\delta^{(m)} F\left(x_{0}\right)$, é um operador causal.

Da (Hip.1) e pelo teorema 3.2.1, F:V $\longrightarrow G^{-}([a, b], X)$ é limitado. Portanto $T$ é limitado. De fato: se $x \in A, A$ limitado e $A \subset V$, então existem $r_{1}>0$ e $r_{2}>0$ tais que

$$
\|x\| \leq r_{1} \text { e }\|F x\| \leq r_{2}, \quad \text { para todo } x \in A .
$$

Logo,

$$
\|T x\|=\|x+k F x\| \leq\|x\|+S V\left[K^{\prime}\right]\|F x\| \leq r_{1}+S V\left[K^{\prime}\right] r_{2} .
$$

A (Hip.2) nos garante a existência de $\delta F$, e esta por sua vez implica a existência de $\delta T$. De fato,

$$
\begin{aligned}
\delta F\left(x_{0}\right)[x](t) & =\lim _{\lambda \rightarrow 0} \frac{F\left(x_{0}+\lambda x\right)(t)-F\left(x_{0}\right)(t)}{\lambda} \\
& =\lim _{\lambda \rightarrow 0} \frac{f\left(t, x_{0}(t)+\lambda x(t)\right)-f\left(t, x_{0}(t)\right)}{\lambda} \\
& =\delta_{2} f\left(t, x_{0}(t)\right)[x](t) .
\end{aligned}
$$


Assim,

$$
\begin{aligned}
\delta T\left(x_{0}\right)[x](t) & =\lim _{\lambda \rightarrow 0} \frac{T\left(x_{0}+\lambda x\right)(t)-T\left(x_{0}\right)(t)}{\lambda} \\
& =\lim _{\lambda \rightarrow 0} \frac{x_{0}(t)+\lambda x(t)+k F\left(x_{0}+\lambda x\right)(t)-x_{0}(t)-k F\left(x_{0}(t)\right.}{\lambda} \\
& =x(t)+k \lim _{\lambda \rightarrow 0} \frac{F\left(x_{0}+\lambda x\right)(t)-F\left(x_{0}\right)(t)}{\lambda} \\
& =x(t)+k F\left(x_{0}\right)[x](t) . k \delta F\left(x_{0}\right)[x](t)
\end{aligned}
$$

Logo,

$$
\delta T\left(x_{0}\right)[x]=x+k F\left(x_{0}\right)[x], x+k \delta F\left(x_{0}\right)[x](t)
$$

isto é,

$$
\delta T=I+k F . I+k \delta \digamma
$$

Por outro lado, sendo $F$ limitado e existindo $\delta F$, o teorema 3.1.4. implica que $F$ é analítico e portanto existe $\delta^{(m)} F$ para todo $m$. Para $m=2$, temos:

$$
\begin{aligned}
\delta^{(2)} T\left(x_{0}\right)\left(x_{1}, x_{2}\right) & =\lim _{\lambda \rightarrow 0} \frac{\delta T\left(x_{0}+\lambda x_{2}\right)\left[x_{1}\right]-\delta T\left(x_{0}\right)\left[x_{1}\right]}{\lambda} \\
& =\lim _{\lambda \rightarrow 0} \frac{x_{1}+k \delta F\left(x_{0}+\lambda x_{2}\right)\left[x_{1}\right]-x_{1}-k \delta F\left(x_{0}\left[x_{1}\right]\right.}{\lambda} \\
& =k \lim _{\lambda \rightarrow 0} \frac{\delta F\left(x_{0}+\lambda x_{2}\right)\left[x_{1}\right]-\delta F\left(x_{0}\right)\left[x_{1}\right]}{\lambda} \\
& =k \delta^{(2)} F\left(x_{0}\right)\left(x_{1}, x_{2}\right) .
\end{aligned}
$$

Indutivamente chegamos a:

$$
\delta^{(m)} T= \begin{cases}I+k \delta F & \text { se } m=1 \\ k \delta^{(m)} F & \text { se } m>1\end{cases}
$$

Da (Hip.3) concluimos que $\delta T\left(x_{0}\right)$ é invertível numa vizinhança de $x_{0}$, pois

$$
\|k \delta F\| \leq\|k\|\|\delta F\| \leq S V[K] \frac{1}{S V[K]}=1 .
$$


Acabamos assim de mostrar que estamos nas hipóteses do teorema de inversão de operadores analíticos em espaços de Banach (teorema 3.1.6.).

Para aplicar a fórmula de inversão desse teorema, precisamos encontrar $H^{-1}$, onde

$$
H=\delta T\left(x_{0}\right)=I+k \delta F\left(x_{0}\right)
$$

Obteremos $H^{-1}$, resolvendo a equação

$$
H x=u
$$

Procurando $H^{-1}$ :

Pelo teorema de representação integral (teorema 2.3.3.), existe $\bar{h}_{1} \in G_{0}^{\sigma} \cdot S V^{u}\left([a, b]^{2}, L(X)\right)$ tal que

$$
\delta F\left(x_{0}\right)[x](t)=\int_{a}^{t} d_{s} \bar{h}_{1}(t, s) x(s)
$$

com

$$
\bar{h}_{1}(t, s) \bar{x}=\delta F\left(x_{0}\right)\left[\chi_{(a, t]} \bar{x}\right](t) \text { e }\|\delta F\|=S V\left[\bar{h}_{1}\right] .
$$

Assim, $(H)$ fica

$$
x(t)+\int_{a}^{t} d_{s} K(t, s) \int_{a}^{s} d_{\sigma} \bar{h}_{1}(t, \sigma) x(\sigma)=u(t) .
$$

Pelo teorema de Bray [12]:

$$
x(t)+\int_{a}^{t} d_{s} \int_{a}^{s} d_{\sigma} K(t, \sigma) \bar{h}_{1}(t, \sigma) x(\sigma)=u(t) .
$$

Então,

$$
x(t)+\int_{a}^{t} d_{s} h_{1}(t, s) x(s)=u(t)
$$


com

$$
h_{1}(t, s)=\int_{s}^{t} d_{\sigma} K(t, \sigma) \bar{h}_{1}(\sigma, s) .
$$

Em [1, Proposição 3.34 (b)] Arbex mostrou que

$$
K, \overline{h_{1}} \in G_{0}^{\sigma} \cdot S V^{u}\left([a, b]^{2}, L(X)\right) \Longrightarrow h_{1} \in G_{0}^{\sigma} \cdot S V^{u}\left([a, b]^{2}, L(X)\right)
$$

Além disso,

$$
C\left(h_{1}, P\right) \leq C(K, P) \cdot S V\left[\overline{h_{1}}\right] .
$$

Mas, segundo a (Hip.1) temos que existe $P \in \mathbb{P}[a, b]$ tal que $C(K, P)<1$, e da (Hip.3), $S V\left[\overline{h_{1}}\right]=\left\|\delta F\left(x_{0}\right)\right\| \leq 1$. Portanto,

$$
C\left(h_{1}, P\right) \leq 1 \text {. }
$$

Logo, pelo teorema 2.3.2.,

$$
H^{-1} u(t)=u(t)-\int_{a}^{t} d_{s} R_{1}(t, s) u(s)
$$

onde

$$
R_{1}=I+\sum_{n=1}^{\infty}(-1)^{n} h_{1}^{(n)}, \quad h_{1}^{(1)}=h_{1} \quad e h_{1}^{(m+1)}(t, s)=\int_{s}^{t} d_{\sigma} h_{1}(t, s) h_{1}^{(m)}(\sigma, s) .
$$

Por outro lado, pelo teorema de representação integral (teorema 2.3.3.), temos que para $m>1$, existe $h_{m} \in G_{0}^{\sigma} \cdot S V^{u}\left([a, b]^{m+1}, L_{m}(X)\right)$ tal que $\delta^{(m)} F\left(v_{0}\right)\left(Q_{j_{1}}, \ldots, Q_{j_{n}}\right)[u]^{m}(t)=\int_{a}^{t} d_{s_{m}} \cdots \int_{a}^{t} d_{s_{1}} h_{m}\left(t, s_{1}, \ldots, s_{m}\right) Q_{j_{1}} u\left(s_{1}\right), \cdots, Q_{j_{n}} u\left(s_{m}\right)$, onde

$$
h_{m}\left(t, s_{1}, \ldots, s_{m}\right)\left(\bar{v}_{1}, \ldots, \bar{v}_{m}\right)=\delta^{(m)} F\left(v_{0}\right)\left(\chi_{\left(a_{1}, t\right]} \bar{v}_{1}, \ldots, \chi_{\left(a_{m}, t\right]} \bar{v}_{m}\right)(t)
$$

E assim, conseguimos expressar todos os termos da série de potências em forma de integrais, conforme a nosso propósito. 


\section{Referências Bibliográficas}

[1] S. E. Arbex. Equações integrais de Volterra-Stieltjes com núcleos descontínuos. PhD thesis, IME-USP, Brasil, 1976.

[2] S. Berberian. Regulated functions: Bourbaki's alternative to Riemann integral. Amer. Math. Monthly, 86, 1979.

[3] I. K. Daugavet and I. Bèsler. Approximation of nonlinear operators by Volterra polynomials. Amer. Math. Soc. Transl. (2), 155, 1993.

[4] R. J. P. de Figueiredo and G. R. Chen. Nonlinear feedback control systems. An operator theory approach. Academic Press, Inc., Boston, 1993.

[5] C. A. Desoer and Vidyasagar. Feedback systems: input-output properties. Electrical Science. Academic Press [Harcourt Brace Jovanovich, Publishers], 1975.

[6] O. Diekmann, M. Gyllenberg, and H. Thieme. Perturbing semigroups by solving Stieltjes renewal equations. Differential Integral Equations, 6(1):155-181, 1993.

[7] O. Diekmann, M. Gyllenberg, and H. Thieme. Perturbing evolutionary systems by step responses and cumulative outputs. Differential Integral Equations, $8(5): 1205-1244,1995$. 
[8] M. Fréchet. Sur les fonctionnelles bilinéaires. Trans. Amer. Math. Soc., 16:215$234,1915$.

[9] M. Gowurin. Über die Stieltjes integration abstrakter funktionen. Fund. Math., 27:254-268, 1936.

[10] E. Hille and R. S. Phillips. Functional analysis and semi-groups, volume 31. rev. ed. American Mathematical Society Colloquium Publications, Providence, 1957.

[11] C. S. Hönig. The abstract Riemann-Stieltjes integral and its applications to linear differential equation with generalized boundary conditions. Notas do IME-USP, Série Matemática, No. 1, SP-Brasil, 1971.

[12] C. S. Hönig. Volterra-Stieltjes Integral Equations. North-Holland Publishing Company-Amsterdam, 1975.

[13] C. S. Hönig. Volterra-Stieltjes integral equations. In Functional differential equations and bifurcation (Proc. Conf., Inst. Ciênc. Mat. São Carlos, Univ. São Paulo, São Carlos 1979), volume 799 of Lecture Notes in Math., pages 173-216. Springer, Berlin, 1980.

[14] C. S. Hönig. Équations intégrales généralisées et applications. In Harmonic analysis: study group on translation-invariant Banach spaces, Exp. No. 5, Publ. Math. Orsay 83, 1. Univ. Paris XI, Orsay, 1983.

[15] C. S. Hönig. Nonlinear Volterra-Stieltjes integral equations. In Seminario Brasileiro de Análise, volume 22, pages 483-486, 1985.

[16] V. Istrăţescu. Fixed Point Theory. An Introduction, volume 7 . D. Reidel Publishigg Company, London:England, 1988. 
[17] H. S. Kalterborn. Linear functional operations on functions having discontinuities of the firt king. Bull. Amer. Math. Soc., 40, 1934.

[18] M. Morse and W. Transue. The Fréchet variation and a generalization for multiple Fourier series of the Jordan test. Rivista Mat., 1:3-18, 1950.

[19] M. Morse and W. Transue. The Fréchet variation, sector limits, and left decompositions. Canadian J. Math., 2:344-374, 1950.

[20] I. Muntean. Classifications of some sets of real functions on a vector space. Studia Univ. Babeş-Bolyai Math., 32(1):60-70, 1987.

[21] M. Z. Nashed. Differentiability and related properties of nonlinear operators: Some aspects of the role of differentials in nonlinear functional analysis. In Nonlinear Functional Anal. and Appl. (Proc. Advanced Sem., Math. Res. Center, Univ. of Wisconsin, Madison, Wis., 1970), pages 103-309, New York, 1971. Academic Press.

[22] D. Pisanelli. Sull'invertibilità degli operatori analitici negli spazi di Banach. Boll. Un. Mat. Ital., 3(19):110-113, 1964.

[23] J. C. Prandini. Extensions of the representation theorems of Riesz and Fréchet. Mathematica Bohemica, 118(3):297-312, 1993.

[24] F. Riesz. Les opérations fonctionnelles linéares. Ann. Sci. Ésc. Norm. Sup., 31(3):2-14, 1914.

[25] M. Schetzen. The Volterra and Wiener theories of nonlinear systems. A WileyInterscience Publication. John Wiley \& Sons, New York-Chichester-Brisbane, 1980. 